\title{
TRA2A-induced upregulation of LINC00662 regulates blood-brain barrier permeability by affecting ELK4 mRNA stability in Alzheimer's microenvironment
}

\section{Qianshuo Liu}

China Medical University

\section{Lu Zhu}

China Medical University

Xiaobai Liu

Shengjing Hospital of China Medical University

\section{Jian Zheng}

Shengjing Hospital of China Medical University

Yunhui Liu

Shengjing Hospital of China Medical University

\section{Xuelei Ruan}

China Medical University

\section{Shuo Cao}

China Medical University

\section{Heng Cai}

Shengjing Hospital of China Medical University

\section{Zhen Li}

Shengjing Hospital of China Medical University

Yixue Xue ( $\sim$ lqsnb666@126.com )

China medical university

\section{Research}

Keywords: blood brain barrier, Alzheimer's disease, TRA2A, LINC00662, ELK4, SMD pathway

Posted Date: December 6th, 2019

DOI: https://doi.org/10.21203/rs.2.17596/v2

License: (c) (P) This work is licensed under a Creative Commons Attribution 4.0 International License. Read Full License

Version of Record: A version of this preprint was published at RNA Biology on May 6th, 2020. See the published version at https://doi.org/10.1080/15476286.2020.1756055. 


\section{Abstract}

The blood-brain barrier (BBB) plays a pivotal role in maintenance and regulation of the neural microenvironment. The occurrence of BBB disruption is the pathological change of early Alzheimer's disease (AD). RNA-binding proteins and long non-coding RNAs are involved in the regulation of BBB permeability. Our study was performed to demonstrate TRA2A/LINC00662/ELK4 axis in regulating BBB permeability in AD microenvironment. In AB1-42-incubated microvascular endothelial cells (ECs) of BBB model in vitro, TRA2A and LINC00662 were enriched. TRA2A increased the stability of LINC00662 by binding with it. The knockdown of either TRA2A or LINC00662 decreased the BBB permeability via upregulating the expressions of tight junctionrelated proteins. ELK4 was lower expressed in BBB model in vitro in AD microenvironment. LINC00662 mediated the degradation of ELK4 mRNA by SMD pathway. The downregulated ELK4 increased the permeability of BTB by increasing the tight junction-related proteins expressions. TRA2A/LINC00662/ELK4 axis plays a crucial role in the regulation of $B B B$ permeability in $A D$ microenvironment, which may provide a novel target for the therapy of $A D$.

\section{Introduction}

Alzheimer's disease (AD), as the most common cause of dementia, seriously affects human health and quality of life ${ }^{[1]}$. At present, since the pathogenesis of $A D$ remains unclear, the existing treatment programs are not effective $e^{[2]}$. The occurrence of blood-brain barrier (BBB) disruption is the pathological change of early AD. The increase of BBB permeability aggravates the degeneration of blood vessels and neurons ${ }^{[3,4]}$. Therefore, function changes of $B B B$ in $A D$ have been a great challenge for identification of pathogenesis and treatments ${ }^{[5]}$. $B B B$ is composed of cerebral microvascular endothelial cells (ECs), pericytes, extracellular matrix and podocytes of perivascular astrocytes ${ }^{[6]}$. The tight junctions between adjacent ECs play a critical role in maintaining BBB integrity. It has been reported that BBB disruption in $A D$ mainly reduces $A \beta$ clearance ${ }^{[4]}$, allowing influx into the brain of neurotoxic blood-derived debris, cells, and microbial pathogens, and is associated with inflammatory and immune responses, which can initiate multiple pathways of neurodegeneration ${ }^{[7]}$.

RNA-binding proteins (RBPs) are a class of proteins that bindings to specific RNA. RBPs can influence the transcription process from alternative splicing, polyadenylation, and nuclear export to cytoplasmic localization, stability, and translation. It has been reported that RBPs, such as Embryonic lethal abnormal vision like protein family (ELVAL), TAR DNA-binding protein 43 (TDP43), eukaryotic initiation factor 2 (elF2a)W2, play pivotal regulatory roles in the development of neurodegenerative diseases including $A D^{[8-10]}$. TRA2A is located at $7 \mathrm{p} 15.3$ and plays an important role in the splicing regulatory process of pre-mRNA ${ }^{[11]}$. TRA2A is highly expressed in the neurodegenerative disease, fragile X-associated tremor/ataxia syndrome (FXTAS). TRA2A aggregates in FXTAS mouse models and in post-mortem human samples, which indicates TRA2A is associated with FXTAS occurrence ${ }^{[12]}$. TRA2A promotes chemotherapy drugs resistance and tumor progression in triplenegative breast cancers via regulating alternative splicing ${ }^{[13]}$. TRA2A is highly expressed in glioma and promotes malignant biological behavior of glioma ${ }^{[14]}$. Additionally, RBPs mediated regulation of vascular function involves with long non-coding RNAs (IncRNAs) ${ }^{[15]}$.

LncRNAs are a group of non-coding RNA transcripts comprising more than 200 nucleotides and lacking apparent open reading frames. LncRNAs have been shown to play important roles in biological processes on the 
transcriptional and post-transcriptional levels, regulating gene transcription, pre-mRNA processing, mRNA stability, protein translation, etc. LncRNAs may provide novel approaches for early diagnosis and treatments of $A D^{[16]}$. LINC00662 is located at $19 q 11$ and relates to tumorigenesis ${ }^{[17,18]}$. However, the regulatory role and potential mechanisms of RBPs and LncRNAs affecting the function of ECs have not been investigated.

ETS-domain protein 4 (ELK4) (also known as SAP1) is a member of the Ets transcription factor family. As a cofactor of serum inflammatory factors, ELK4 participates in inflammatory response ${ }^{[19]}$, insulin dependence regulation ${ }^{[20]}$, etc. In gliomas, down-regulation of ELK4 can decrease the expression of $\mathrm{Mcl}-1$ and induce the sensitivity of tumor cells to apoptosis ${ }^{[21]}$. ELK4 as a transcriptional regulator may be involved in the expression of neuronal plasticity regulatory genes following sleep deprivation ${ }^{[22]}$. By searching the bioinformatics database RepeatMasker, we noticed that there is a Alu element in ELK4's 3'-UTR region. We also predicted that the putative binding site exists between LINC00662 and ELK4 3'-UTR region using bioinformatics software IntaRNA.

STAU1-mediated mRNA decay (SMD) pathway is an mRNA degradation pathway that regulates biological processes. SMD usually occurs following a normal translation termination event as a means to fine-tune the levels of transcripts harboring a Stau1 binding site (SBS). SBSs can be formed by base-pairing between an Alu element within the $3^{\prime} U T R$ of an SMD target and another Alu element within a IncRNA ${ }^{[23]}$. SMD pathway plays an important role in tumorigenesis ${ }^{[15]}$ and cell differentiation ${ }^{[24]}$. Studies have shown that STAU1 is highly expressed in Spinocerebellar ataxia type 2 (SCA2), a neurodegenerative disease model, and involved in abnormal RNA metabolism ${ }^{[25]}$. Thus, STAU1 may become a therapeutic target for neurodegenerative diseases.

In present study, endogenous expressions of TRA2A, LINC00662 and ELK4 in ECs after A $\beta_{1-42}$ incubation were detected, and interactions among them were analyzed to confirm the regulatory mechanisms for BBB permeability. Our study may provide a new target for AD treatment regard of BBB.

\section{Material And Methods}

\section{Cell cultures}

The human cerebral microvascular endothelial cell line hCMEC/D3 (ECs) were provided by Dr. Couraud (Institut Cochin, Paris, France). Human brain vascular pericytes (HBVP) and normal human astrocytes (NHA) were purchased from the Sciencell Research Laboratories (Carlsbad, CA, USA). NHA and HBVP applied in this study were limited with passage below 12. ECs (passage 28-32), HBVP (passage 10-12) and NHA (passage 10-12) were cultured as described previously ${ }^{[26]}$. Human embryonic kidney 293 (HEK293T) cells were purchased from Shanghai Institutes for Biological Sciences Cell Resource Center and the cell culture has been previously detailed $^{[27]}$. All cells were maintained at $37^{\circ} \mathrm{C}, 5 \% \mathrm{CO} 2$, in a humidified atmosphere. $A \beta_{1-42}$ was manufactured by Sigma-Aldrich (St. Louis, MO, USA). $A \beta_{1-42}$ was first dissolved in dry DMSO $(2 \mathrm{mmol} / \mathrm{L})$ and stored at $-20^{\circ} \mathrm{C}$. $2 \mathrm{mmol} / \mathrm{L} \mathrm{A} \beta_{1-42}$ in DMSO was diluted into $200 \mu \mathrm{mol} / \mathrm{L}$ in cold Opti-MEM media and incubated at $4^{\circ} \mathrm{C}$ for 24 hours. Cells were pre-incubated with $A \beta_{1-42}$ at a concentration of $5 \mu \mathrm{mol} / \mathrm{L}$ for 48 hours following Liu et al [26].

\section{In vitro BBB model establishment}


In vitro co-culturing BBB models were established as described previousely ${ }^{[26]}$. First, after pericytes were cultured ( $\left.2 \times 10^{5} \mathrm{cells} / \mathrm{cm}^{2}\right)$ on the lower chamber of Transwell inserts $(0.4 \mu \mathrm{m}$ pore size; Corning, NY, USA) overnight, hCMEC/D3 cells were subsequently placed on the upper chambers of Transwell inserts. NHA $\left(2 \times 10^{5}\right.$ cells $\left./ \mathrm{cm} 2\right)$ were seeded onto the 6-well culture plate and cultured for 2 days before adding ECs inserts.

\section{Real-time PCR assays}

Nanodrop Spectrophotometer (ND-100, Thermo Scientific, Waltham, MA) was applied to determine the RNA concentration and quality. The expression levels of TRA2A (NM_001282757), LINC00662 (NR_027301) and ELK4 (NM_001973.4) were detected by One-Step SYBR PrimeScript RT-PCR Kit (Perfect Real Time; Takara Bio, Inc., Kusatsu, Japan). Relative expression values were calculated using the relative quantification $\left(2^{-} \triangle \triangle \mathrm{Ct}\right)$ method. Primers were shown in Table A1.

\section{Cell transfections}

Silencing plasmid of LINC00662 was ligated into LV10 (U6/RFP\&Puro) vector (GenePharma, Shanghai, China) to construct the shLINC00662 plasmid. Short-hairpin RNA directed against human TRA2A gene, STAU1 gene, UPF1 gene and ELK4 gene was ligated into the pGPU6/GFP/Neo vector (GenePharma) to construct shTRA2A, shSTAU1, shUPF1, and shELK4 plasmid, respectively. The human ELK4 gene coding sequence was ligated into pIRES2 vector (GenScript, Piscataway, NJ, USA) to construct the ELK4 overexpression plasmid. The respective no-targeting sequences were used as NCs. The ECs were stable transfected via LTX and Plus reagent (Life Technologies, Carlsbad, CA, USA). The stable transfected cells were selected using G418 (0.4 mg/ml) (SigmaAldrich, St. Louis, MO, USA) or puromycin $(1 \mu \mathrm{g} / \mathrm{ml})$ (Sigma-Aldrich). Sequences of shTRA2A, shLINC00662, shELK4, shSTAU1, shUPF1 were shown in Table A2. The silencing and over-expressions efficiency were measured by qRT-PCR. The transfected efficiency of TRA2A, LINC00662 and ELK4 were shown in Figure. S-A-C, $\mathrm{H}$, I. For co-transfection of shTRA2A and shLINC00662 (shTRA2A+shLINC00662), shLINC00662 was transiently transfected into $A \beta_{1-42}$-incubated ECs, which stably transfected shTRA2A with lipofectamine 3000 reagent. After 48 hours, the transiently transfected cells were obtained. Other co-transfected ECs were established in the same way.

\section{Transendothelial electric resistance (TEER) assays}

A millicell-ERS apparatus (Millipore, Billerica, MA, USA) was used to perform TEER assays after in vitro BBB models constructed. Each measurement was placed in room temperature for 30 minutes, and TEER values was recorded. TEER values were measured after the medium exchange. Background electrica resistances were subtracted before the final resistances were calculated. TEER values $\left(\Omega \cdot \mathrm{cm}^{2}\right)$ is electric resistance multiplied by the effective surface area of the transwell insert.

\section{Horseradish peroxidase (HRP) flux assays}


In vitro BBB models were constructed and $1 \mathrm{ml}$ of serum-free EBM-2 medium containing $10 \mu \mathrm{g} / \mathrm{mL} \mathrm{HRP}(0.5$ $\mathrm{mmol} / \mathrm{L}$, Sigma-Aldrich) culture medium was added into the upper champer of the transwell system. 1 hour later, $5 \mu \mathrm{L}$ of culture medium in the lower chamber was collected and the HRP content of the samples was detected by tetramethylbenzidine colorimetry approach. The final HRP value was expressed as $\mathrm{pmol} / \mathrm{cm}^{2} / \mathrm{h}$.

\section{Western blot assays}

The cell lysates were extracted from ECs. Total proteins were extacted with RIPA buffer (Beyotime Institute of Biotechnology, Jiangsu, China) supplemented with protease inhibitors $(10 \mathrm{mg} / \mathrm{mL}$ aprotinin, $10 \mathrm{mg} / \mathrm{mL}$ phenylmethylsulfonyl fluoride[PMSF], and $50 \mathrm{mM}$ sodium orthovanadate) and centrifuged at $17,000 \times \mathrm{g}$ for 30 minutes at $4^{\circ} \mathrm{C}$. Equal amounts of proteins were further separated using SDS-PAGE and then transferred to polyvinylidene fluoride (PVDF) membranes (Millipore, Shanghai, China). Membranes were blocked to avoid nonspecific bindings in Tris-buffered saline-Tween (TBST) containing $5 \%$ fat-free milk for 2 hours and subsequently incubated with primary antibodies (shown in Table A4) at $4^{\circ} \mathrm{C}$ overnight. After three washes with TBST, membranes were incubated with the corresponding secondary antibody at a 1:10000 dilution at room temperature for 2 hours. Immunoblots were visualized by enhanced chemiluminescence (ECL kit, Santa Cruz Biotechnology) after washes. All the protein bands were scanned by Chem Imager 5500 V2.03 software and the integrated density values (IDVs) were calculated utilizing FluorChem 2.0 software.

\section{Immunofluorescence assays}

Cells were fixed by $4 \%$ paraformaldehyde for 20 minutes, and permeated in phosphatebuffered saline (PBS) containing $0.2 \%$ Triton $X-100$ for 10 min. Next, cells were blocked by $5 \%$ bovine serum album (BSA) in PBS for 2 hours at room temperature, and incubated with primary antibodies (anti-ZO-1, 1:50, Life Technologies; antioccludin, 1:50, Life Technologies; anti-claudin-5, 1:50, Life Technologies;) respectively at $4^{\circ} \mathrm{C}$ overnight. After three washes with PBS, cells were incubated with fluorophore-conjugated secondary antibodies for 2 hours. DAPI were applied to observe cell nuclei. The staining was oberservrd by immunofluorescence microscope (Olympus, Tokyo,Japan).

\section{Chromatin immunoprecipitation assays}

Chromatin immunoprecipitation (ChIP) kit (Cell Signaling Technology, Danvers, MA, USA) was used for ChIP assays following the manufacture's description. Briefly, cells were crosslinked with $1 \%$ formaldehyde and collected in lysis buffer. Chromatin was then digested with Micrococcal Nuclease. Immunoprecipitates were incubated with $3 \mu \mathrm{g}$ anti-ELK4 antibody (Santa Cruz, USA) or normal rabbit IgG and incubated with Protein $\mathrm{G}$ agarose beads at $4^{\circ} \mathrm{C}$ overnight with gentle shaking while $2 \%$ lysates were used as input reference. DNA crosslink was reversed with $5 \mathrm{M} \mathrm{NaCl}$ and proteinase $\mathrm{K}$ and purified. Immunoprecipitation DNA was amplified by PCR using their spcific primers. In each PCR reaction, the corresponding inputs were taken in parallel for PCR validation. Primers used for ChIP PCR are shown in Table A3. 


\section{Human IncRNA and RNA microarrays}

LncRNA and RNA analysis, sample preparation, and microarray hybridization were performed by Kangchen Biotech (Shanghai, China).

\section{Reporter vector construction and dual-luciferase reporter assays}

The putative LINC00662 binding regions in the ELK4 gene were amplified by PCR and cloned into downstream of pmirGLO dual-luciferase vector (Promega, Madison, WI, USA) to form the wide-type plasmid (ELK4-3'UTR-Wt) (GenePharma). In the same way, the binding sequences were mutated as indicated to form the mutant-type plasmid (ELK4-3'UTR-Mut). The pmirGLO vector constructed with either 3'-UTR fragments or mutation of 3'-UTR fragments, and LINC00662 or LINC00662-NC were cotransfected into HEK293T cells in 24-well plates using Lipofectamine 3000 . ECs were harvested 48 hours after transfection and analyzed by luciferase assay using the Dual-Luciferase Reporter Assay System (Promega). The relative luciferase activity was expressed as the ratio of firefly luciferase activity to renilla luciferase activity.

Human genomic DNA was used to amplify different promoter fragments, subcloned into pGL3-Basic-Luciferase vector (Promega) containing a firefly luciferase reporter gene and verified by DNA sequencing. Human full-length ELK4 was constructed in pEX3 (pGCMV/MCS/Neo) plasmid vector (GenePharma). HEK293T cells were cotransfected with the pGL3 vector of ZO-1, occludin, and claudin-5 with either full-length promoter regions (or deleted promoter regions) and pEX3-ELK4 (or pEX3 empty vector) using Lipofectamine 3000. Relative luciferase activity was analyzed as described previously.

\section{RNA immunoprecipitation (RIP) assays}

EZ-Magna RNA-binding protein immunoprecipitation kit (Millipore, USA) was used according to the manufacture's protocol. Whole cell lysate was incubated with human $5 \mu \mathrm{g}$ human anti-Ago2 antibody, or NC normal mouse lgG. Furthermore, purified RNA was extracted and applied to qRT-PCR to demonatrate the presence of the binding targets.

\section{RNA pull-down assays}

Biotin-labelled, full length LINC00662, or antisense RNA was prepared with the Biotin RNA Labeling Mix (GenePharma, Shanghai, China) and transfected into ECs. Biotinylated RNAs were treated with RNase-free DNase I and purified. RNA-protein complexes were isolated by streptavidin agarose beads (Invitrogen, Shanghai, China) and washed three times. The retrieved proteins were detected using a standard western blotting technique with GAPDH as the control.

\section{Nascent RNA capture}


Nascent RNAs was detected using Click-iTß Nascent RNA Capture Kit (Thermo Fisher Scientific,USA) according to the manufacture's protocol. Briefly, nascent RNAs were marked with $0.2 \mathrm{mM} 5$-ethymyl uridine (EU) and the EUnascent RNA was captured on magnetic beads for subsequent qRT-PCR.

\section{mRNA stability assays}

In order to inhibit the de novo RNA synthesis, $5 \mathrm{ug} / \mathrm{ml}$ actinomycin D (ActD, NobleRyder, China) was added into ECs culture medium. Total RNA was extracted at $0,2,4,6,8,10 \mathrm{~h}$ and its concentrations were easured by qRTPCR. The half-life of RNA was determined by its level at certain point of time compared with time zero.

\section{Satistical analysis}

Statistical analysis was performed with GraphPad Prism v7.00 (GraphPad Software, La Jolla, CA, USA) software. Data was described as mean \pm standard deviation (SD). All differences were analyzed by SPSS 18.0 statistical software with the Student's t-test (two tailed) or one-way ANOVA. Differences were considered as statically significant when $P<0.05$.

\section{Results}

\section{TRA2A was highly expressed in $A \beta_{1-42}$-incubated ECs, and knockdown of TRA2A attenuatd BBB permeability in $\mathrm{AD}$ microenvironment}

To verify the role of TRA2A in BBB permeability in AD microenvironment, TRA2A levels in $A \beta_{1-42}$-incubated ECs was detected by qRT-PCR and Western blot. As shown in Figure. 1-A,B, TRA2A mRNA and protein levels in $A \beta_{1-}$

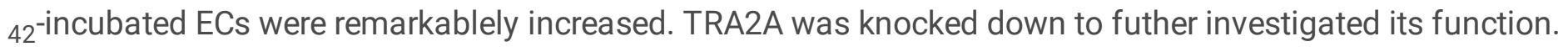
When the BBB model in vitro was successfully constructed using stable TRA2A knockdown ECs in AD microenvironment, TEER values, HRP flux were detected to analyze the integrity and permeability of BBB, respectively. Compared with shNC group, TEER in shTRA2A group increased (Figure. 1-C), HRP flux attenuated significantly (Figure.1-D), indicating that TRA2A konckdown decreased BBB permeability in AD microenvironment. ZO-1, occludin and claudin-5 are members of tight junction-related proteins. We focused on the three proteins to investigate the mechanism of action of TRA2A on BBB permeability. The expression of ZO1 , occludin and claudin-5 were further detected. As shown in Figure. 1-E,F, knockdown of TRA2A significantly induced ZO-1, occludin and claudin- 5 mRNA and protein expression levels in A $\beta_{1-42}$-incubated ECs. Moreover, consistent with the western blot results, immunofluorescence showed that TRA2A knockdown increased the expression of ZO-1, occludin and claudin-5, with discontinuous distribution to relatively continuous distribution on the boundaries of $A \beta_{1-42}$-incubated ECs. 


\section{LINC00662 was up-regulated in $A \beta_{1-42}$-incubated ECs, and knockdown of LINC00662 decreased BBB permeability in AD microenvironment}

To investigate IncRNA that involved in TRA2A-mediated regulation on BBB permeability, we used IncRNA microarray. It was found LINC00094, LINC00662 and FIRRE were significantly downregulated in ECs transfected with shTRA2A plasmid (Figure 2-A). Further, the IncRNAs level in shTRA2A ECs was investigated by qRT-PCR. As Figure 2-B shows, LINC00662 expression was remarkablely attenuated in A $\beta_{1-42}$-incubated ECs treated with shTRA2A. Moreover, LINC00662 was upregulated in A $\beta_{1-42}$-incubated ECs (Figure 2-C). TEER value in shLINC00662 group increased significantly (Figure. 2-D), HRP flux attenuated significantly (Figure. 2-E) compared with shNC group. The mechanistic studies showed, the mRNA and protein levels of ZO-1, occludin, and claudin-5 in A $\beta_{1-42}$-incubated ECs were promoted after LINC00662 knockdown (Figure. 2-F, G). Immunofluorescence confirmed that downregulation of LINC00662 induce ZO-1, occludin and claudin-5, with more continuous distribution on the boundaries of ECs (Figure. 2-H).

\section{LINC00662 was involved in TRA2A-mediated regulation of BBB permeability}

As shown in Figure 3-A, the expression level of LINC00662 in shTRA2A group was significantly lower than that in shNC group using qRT-PCR. To explore the correlation between TRA2A and LINC00662, RIP assays and RNA pull-down assays were used to confirm that TRA2A binds to LINC00662 directly. As shown in Figure. 3-B, the enrichment of LINC00662 in anti-TRA2A group was higher than anti-IgG group. The level of TRA2A captured by Anti-LINC00662 group was significantly higher than that in Anti-antisense group (Figure. 3-C). Moreover, qRTPCR demonstrated that novel LINC00662 level in shNC group and shTRA2A group is not statistically significant (Figure. 3-D). As shown in Figure. 3-E, the half-life of LINC00662 in shTRA2A group was significantly impaired than that in shNC group. LINC00662 knockdown enhanced the increase in TEER values (Figure. 3-F) and the impairment in HRP flux (Figure. 3-G) caused by TRA2A knockdown. LINC00662 knockdown magnified the increase in ZO-1, occludin, claudin-5 expression caused by TRA2A knockdown (Figure. 3-H). These results indicated that TRA2A increased BBB permeability by stabilizing LINC00662.

\section{ELK4 was down-regulated in $A \beta_{1-42}$-incubated ECs, and attenuated $\mathrm{BBB}$ permeability by regulating $\mathrm{ZO}-1$, occludin, claudin-5}

When LINC00662 was knockdown in A $\beta_{1-42}$-incubated ECs, microarray analysis showed ZEB1, ELK4 and ART1 are three most abundant (Figure. 4-A). The increase of the ELK4 expression investigated by qRT-PCR was the most significant (Figure. 4-B). ELK4 protein levels was highly expressed in shLINC00662 group (Figure 4-C). As shown in Figure. 4-D,E, the mRNA and protein expression levels of ELK4 in A $\beta_{1-42}$-incubated ECs was lower than 
that in control group. To verify the role of ELK4 in BBB permeability in AD microenvironment, we knocked down and overexpressed ELK4 to elucidate its function. As shown in Figure. 4-F,G, compared with ELK4-NC group, TEER in ELK4 group increased significantly, HRP flux decreased significantly; compared with shELK4-NC group, TEER in shELK4 group decreased significantly and HRP flux increased significantly. Moreover, ZO-1, occludin, claudin- 5 mRNA and protein exhibited higher expression level in ELK4 group, and ELK4 knockdown inhibited ZO-1, occludin and claudin-5 expression (Figure. 4-H,I). Subsequent immunofluorescence showed that ZO-1, occludin and claudin -5 were upregulated in ELK4 group, which exhibited relative continuous distribution on the boundaries of $A \beta_{1-42}$-incubated ECs. ELK4 knockdown leaded to the opposite result (Figure. $4 \mathrm{~J}$ ). We further explored the role of TRA2A and LINC00662 on the expression of ELK4. Compared with shTRA2A-

NC+shLINC00662-NC group, shTRA2A+shLINC00662-NC group and shTRA2A-NC+shLINC00662 group exhibited higher expression of ELK4. The expression of ELK4 in shTRA2A+shLINC00662 group was significantly increased compared with shTRA2A+shLINC00662-NC group and shTRA2A-NC+ shLINC00662 group respectively.

\section{LINC00662 down-regulated the expression of ELK4 through SMD pathway, downregulated the expression of ZO-1, occludin and claudin-5, and increased the permeability of BBB in AD microenvironment}

By quiring the bioinformatics database RepeatMasker and IntaRNA, we found that LINC00662 has the putative binding site with the ELK4 3'UTR (Figure. S-E-G). Therefore, we hypothesized that LINC00662 exhibits a negative regulatory effect on ELK4 via SMD pathway. The interaction between LINC00662, ELK4 and STAU1 was detected by dual-luciferase reporter assays, RNA pull down experiments and RIP. To verify our hypothesis, we used dual-luciferase reporter assay to confirm the predicted binding site. The relative luciferase activity in ELK4-3'UTR-Wt+LINC00662 group was significantly reduced compared with ELK4-3'UTR-Wt+LINC00662-NC group, while there was no significant difference between ELK4-3'UTR-Mut+LINC00662 and ELK4-3'UTRMut+LINC00662-NC group (Figure.5-A). RNA pull down experiments also confirmed that LINC00662 bind directly to ELK4 and STAU1, respectively (Figure. 5-B,D). Furthermore, as shown in Figure 5-C,E, RIP confirmed that LINC00662 and ELK4 mRNA bind to STAU1 directly respectively. The relative enrichment of LINC00662 and ELK4 mRNA in anti-STAU1 group was higher than anti-IgG group respectively. In order to confirm that STAU1 participates in the interaction between LINC00662 and ELK4, A $\beta_{1-42}$-incubated ECs was co-tansfected with shSTAU1 and shLINC00662 plasmid. qRT-PCR demonstrated that nacent LINC00662 levels in control group, shSTAU1-NC+shLINC00662-NC group, shSTAU1+shLINC00662-NC group, shSTAU1-NC+shLINC00662 group and shSTAU1+shLINC00662 group are not statistically significant (Figure. 5-F). The half-life of ELK4 mRNA was significantly increased in shLINC00662+shSTAU1 group (Figure. 5-G). ShSTAU1+shLINC00662-NC and shSTAU1-NC+shLINC00662 groups exhibited higher ELK4 expression compared with shSTAU1-

NC+shLINC00662-NC group respectively. The promotive effect was magnified in shSTAU1+shLINC00662 group (Figure. 5-H). To verify the effect of UPF1 on ELK4, ECs was transfected with shUPF1 plasmid. There were no statistical differences in nacent ELK4 mRNA between control group, shNC group and shUPF1 group. The halflife of ELK4 mRNA was significantly increased in shUPF1 group (Figure. S-J). ELK4 protein expression level 
increased significantly in shUPF1 group (Figure. S-K). As shown in Figure. 5-I, J, compared with shLINC00662NC+ELK4-NC group, TEER in shLINC00662+ELK4 group increased and HRP flux decreased significantly. Coknockdown of ELK4 and LINC00662 largely reversed the LINC00662 knockdown induced the increase in TEER

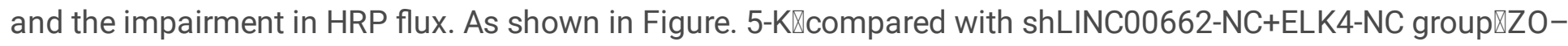
1 , occludin, claudin-5 protein expression was increased in shLINC00662+ELK4 group. Moreover, co-knockdown of LINC00662 and ELK4 largely reversed the promotive effect of ZO-1, occludin, claudin-5 expression in $A \beta_{1-42}$ incubated ECs caused by LINC00662 konckdown.

\section{ELK4 bound to the promoters of ZO-1, occludin and claudin-5 and promoted transcription}

By using the bioinformatics software JASPAR, we predicted that the ZO-1, occludin, claudin-5 promoter might harbor upstream putative binding sites for ELK4. Subsequent dual-luciferase reporter assays and ChIP assays were performed to identify any association between ELK4 and the above promoters. As shown in Figure. 6-A-C, following co-transfection with pEX3-ELK4, ZO-1, occludin, and claudin-5 promoter activities were substantially increased. Subsequently, putative ELK4 binding sites in the promoter reporter constructs were deleted one by one. As shown in Figure 6-A, deletion of the -880 site and the -570 site caused reduction of the ZO-1 promoter activity. Whereas, after the deletion of the -381 site, the promoter activity didn't change significantly. As shown in Figure 6-B, deletion of the -531 site diminished the occludin promoter activity as compared to the full-length occludin promoter reporter construct. As shown in Figure $6 \mathrm{C}$, the construct containing a -153 site and -81 site deletion produced reversion of promoter activity increase. In addition, the DNA binding pattern of ELK4, as determined by ChIP assays, was consistent with the locations of the binding sites on the ZO-1, occludin, and claudin-5 promoters determined in dual-luciferase reporter assay. As shown in Figure. 6D-F, the specific primers for the $-880,-570,-381$ site regions of the ZO- 1 promoter, -531 site region of the occludin promoter and $327,-153,-81$ site regions of the claudin- 5 promoter were used. In ELK4 immunoprecipitates, PCR constructs were observed using primers specific mentioned above and the $3000 \mathrm{bp}$ upstream regions were used as negative control. Collectively, our data indicated that ELK4 exhibit transcriptional promotion of ZO-1, occludin, and claudin -5 by binding to specific sites in these gene's promoters.

\section{Discussion}

This study demonstrated for the first time that RBP-TRA2A and IncRNA-LINC00662 were highly expressed in $A \beta_{1-42}$ incubated ECs, and knockdown of TRA2A or LINC00662 could significantly reduce BBB permeability in AD microenvironment. TRA2A knockdown reduced the stability of LINC00662 and down-regulated its expression; down-regulated LINC00662 attenuated the degradation of ELK4 mRNA through the SMD pathway, thereby increasing the expression of ELK4. Moreover, restoration of ELK4 decreased BBB permeability via transcriptional promotion of ZO-1, occludin, and claudin-5. The TRA2A/LINC00662/ELK4 axis is presented in Figure. 7 schematically.

BBB plays an critical role in maintaining the homeostasis and brain functions of the central nervous system. BBB breakdown contributes to the onset and progression of $A D^{[28,29]}$. It has been found that BBB dysfunction causes diminishment of $A \beta$ clearance, leading to $A \beta$ deposition ${ }^{[30-32]}$. There are two main pathways involved in 
the regulation of BBB permeability: transcellular and paracellular pathway ${ }^{[33]}$. Tight junction-related proteins between ECs play a key role in the regulation of the paracellular pathway. Abnormal expression and distribution of tight junction-related proteins can affect BBB permeability ${ }^{[34]}$. It has been reported that $A \beta$ is toxic to brain ECs via binding to receptor for advanced glycation endproducts (RAGE) and induction of reactive oxygen species (ROS) production, which ultimately leads to disruption of tight junction-related proteins and loss of BBB integrity $^{[35]}$. Granulocyte-macrophage colony-stimulating factor (GM-CSF) triggers human brain microvascular ECs tight junction disassembly by downregulating ZO-1 expression via transcription modulation and claudin-5 expression through the ubiquitination pathway. In AD patients, high GM-CSF levels in the brain parenchyma and cerebrospinal fluid induce blood-brain barrier opening ${ }^{[36]}$. It has also been reported that BBB breakdown caused by lower expression and discontinuous distribution of tight junction-related proteins occurs in other neurodegenerative diseases and age-associated brain changes ${ }^{[37,38]}$. In this study, $A \beta_{1-42^{-i n c u b a t e d ~ h u m a n ~}}$ brain microvascular endothelial cells (hCMEC/D3) were used to simulate the model of BBB in AD microenvironment. The integrity and permeability of BBB were reflected by TEER and HRP flux, and the expression levels and distribution of ZO-1, occludin and claudin-5 were detected to clarify the possible mechanisms of BBB permeability.

Numerous reports have shown that RBPs play an important regulatory role in neurodegenerative diseases. nELAVL proteins are abundant neuron-specific RBPs which have been suggested to regulate various neurological processes and have been linked to neurodegenerative disorders including $A D$ and PD. The formation of $n E L A V L / Y$ RNA complex after binding to non-coding Y RNA in conditions of AD leads to $n E L A V L$ isolation. $n E L A V L$ isolation results in $n E L A V L$ sequestration, redistribution of $n E L A V L$ target binding, and altered neuronal RNA splicing ${ }^{[39]}$. Fused in sarcoma (FUS) gene is a RBP participating in many RNA metabolism pathways, and mutations in FUS contribute to a subset of familial Amyotrophic lateral sclerosis (ALS) cases. Mutant FUS suppresses protein biosynthesis and disrupts the nonsense-mediated decay (NMD) regulation, both of which likely contribute to motor neuron death ${ }^{[40]}$. TRA2A was confirmed to be upregulated in $A \beta_{1-42^{-}}$ incubated ECs. Knockdown of TRA2A attenuated BBB permeability in AD microenvironment by increasing the expression of ZO-1, occludin and claudin-5. RBPs have played an pivotal role in regulating vascular endothelial function. RBP QKI is highly expressed in ECs. QKI directly binds to the mRNA of VE-cadherin and $\beta$ catenin and can promote mRNA translation, maintaining endothelial barrier function ${ }^{[41]}$. RBPs MOV10 and FUS regulate vascular endothelial function by binding to Circular RNAs (circRNAs) ${ }^{[42,43]}$.

LncRNAs play an important role in brain development and neurodegenerative diseases including epigenetic regulation, transcriptional regulation, post-transcriptional regulation, translational regulation ${ }^{[44,45]}$. LncRNAs expression changes in HD are widespread, that many of these result in altered epigenetic gene regulation in diseased neurons, and that contributes to neurodegeneration ${ }^{[46]}$. A novel IncRNA (named 17A) is upregulated in cerebral tissues derived from AD patients and embeds in the human G-protein-coupled receptor 51 gene (GPR51, GABA B2 receptor). The stable expression of 17A in SHSY5Y neuroblastoma cells induces the synthesis of an alternative splicing isoform that abolish GABA B2 intracellular signaling and enhances the secretion of $A \beta$. Thus $17 A$ is directly or indirectly involved in the occurrence and development of $A D^{[47]}$. LncRNA NDM29 is highly expressed in AD. NDM29 expression promotes the molecular processing and the secretion of $A \beta$, as it occurs in $A D^{[48]}$. In our study, LINC00662 was highly expressed in A $\beta_{1-42}$-incubated ECs. Knockdown of LINC00662 decreased $\mathrm{BBB}$ permeability in AD microenvironment by increasing the expression of tight junction-related 
proteins. Accumulated evidences have indicated that LncRNAs regulate vascular endothelial function. LncRNACCL2 positively regulates CCL2 mRNA levels in ECs, which may contribute to endothelial inflammatory changes ${ }^{[49]}$. LncRNA SENCR promotes EC adherens junction integrity through physical association with CKAP4, thereby stabilizing cell membrane ${ }^{[50]}$. LINC00094 affects blood-brain barrier permeability in AD microenvironment by regulating the expression of tight junction-related proteins ${ }^{[34]}$.

Recently, it has been a subject of intense studies that the interaction between RNA-binding proteins and noncoding RNAs regulates vascular endothelial function. We found that TRA2A can increase the stability of LINC00662 and reduce BBB permeability in AD microenvironment. One of the mechanisms is that RBPs alter the stability of non-coding RNAs and regulate vascular endothelial function. For example, Wu et al/[1] have reported that RBP KHDRBS3 binds to circRNA DENND4C, increases the circRNA's stability and upregulates the expression of tight junction-related proteins, thereby reducing the permeability of blood tumor barrier. Li et al[ ${ }^{[5]}$ have reported that RBP ZRANB2 binds to the IncRNA and increases the IncRNA's stability, thereby affecting the functions of vascular ECs. A similar report has been made in AD that RBP HuD associates with and stabilizes the IncRNA BACE1AS, which partly complements BACE1 mRNA (encoding $\beta$-site APP-cleaving enzyme 1). HuD elevates the levels of APP, BACE1, BACE1AS, and promotes the production of APP and the cleavage of its amyloidogenic fragment, $A \beta^{[52]}$.

ELK4 is a member of the Ets transcription factor family. The Ets transcription factor family is a key regulator of many neurodevelopmental events ${ }^{[33]}$. It has been shown that ELK4 not only can directly bind to gene promoter to regulate its transcription, but also can act as a cofactor cooperating with other transcription factors to influence target gene transcription ${ }^{[19,54]}$. ELK4 upregulates $\mathrm{Mcl}-1$ by binding to $\mathrm{Mcl}-1$ promoter and improves its activity, thereby decreasing glioma cells sensitivity to apoptosis ${ }^{[21]}$. In HepG2 cells, ELK4 is a protein through which SIRT7 targets the G6PC promoter, regulates the transcriptional activity of G6PC under glucose deprivation, and participates in the regulation of gluconeogenesis ${ }^{[55]}$. ELK4 is enriched in promoter bindings of genes upregulated in peripheral blood mononuclear cells (PBMC) from non-small cell lung cancer (NSCLC) patients before tumor removal. Thus $邓$ transcriptional promotion function of ELK4 may be involved in the interaction between NSCLC and immunologically important pathways ${ }^{[56]}$. In present study, dual-luciferase gene reporter assays and ChIP assays were used to demonstrate for the first time that transcription factor ELK4 could bind to ZO-1, occludin, claudin -5 promoter and enhance its activity, thereby increasing the expression of ZO-1, occludin, claudin -5 and reducing BBB permeability in AD microenvironment.

Current studies have shown that SMD pathway plays an important role in neurodevelopment and neurological diseases. It has been reported that KLF4 promotes Stau1 recruitment to the $3^{\prime} U T R$ of neurogenesis-associated mRNAs, increasing SMD pathway of these transcripts. The molecular mechanism underlying stability of neurogenesis-associated mRNAs affects neurogenesis and self-renewal in mice ${ }^{[57]}$. In addition, in mouse 3T3-L1 cells, PNRC2 interacts with STAU1 and UPF1 to reduce the stability of KLF2 mRNA and accelerates adipogenesis through SMD pathway ${ }^{[24]}$. We found that knockdown of LINC00662, STAU1 and UPF1 could prolong the half-life of ELK4 improving the stability of ELK4 mRNA and increase the expression of ELK4. Our study proved, for the first time, that LINC00662 promoted ELK4 degradation via SMD pathway in ECs, thus increasing BBB permeability in AD microenvironment. Earlier studies have shown that IncRNAs regulate the function of vascular ECs and tumor cells by SMD pathway ${ }^{[58]}$. For example, SNHG20 reduces FOX1 mRNA 


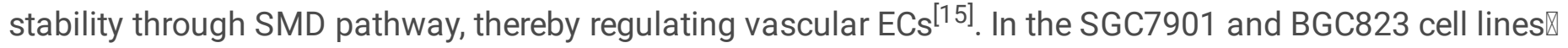
IncRNA TINCR impairment the stability of KLF12 mRNA via SMD pathway, affecting the proliferation and apoptosis of gastric cancer cells ${ }^{[59]}$.

\section{Conclusions}

In conclusion, we studied TRA2A and LINC00662 expression increased and ELK4 expression impaired in A $\beta_{1-42^{-}}$ incubated ECs. TRA2A bound to LINC00662, and LIN00662 acted on ELK4 via SMD pathway. Knockdown of TRA2A reduced the stability of LINC00662, and reduced the degradation of ELK4 mRNA by SMD pathway subsequently. ELK4 promoted transcription by binding to the promoters of ZO-1, occludin and claudin- 5 and further reduced the permeability of blood-brain barrier in AD microenvironment. More significantly, TRA2A, LINC00662 and ELK4 may have a significant therapeutic potential in AD treatment. Our results provide a new mechanistic insight into the pathogenesis of $A D$ and identify novel targets for its treatment.

\section{Declarations}

\section{Ethics approval and consent to participate}

Not applicable.

\section{Consent for publication}

Not applicable.

\section{Availability of data and materials}

Data supporting our findings are found within the manuscript and in the additional files.

\section{Competing interests}

The authors have declared that no competing interest exists.

\section{Funding}

No funding was received.

\section{Authors' Contributions}

YXX contributed to the experiment design and implementation, manuscript draft, and data analysis. QSL contributed to the experiment implementation and data analysis. YHL conceived or designed the experiments. QSL, LZ, XBL, and JZ performed the experiments. LZ, XLR, SC, HC and ZL analyzed the data. QSL conceived or 
designed the experiments, performed the experiments, and wrote the manuscript. All authors read and approved the final manuscript.

\section{Acknowledgement}

Not Applicable.

\section{References}

[1] R. Anand, K. D. Gill, A. A. Mahdi. Therapeutics of Alzheimer's disease: Past, present and future, Neuropharmacology, 76 Pt A (2014) 27-50.

[2] L. B. Gao, X. F. Yu, Q. Chen, D. Zhou. Alzheimer's Disease therapeutics: current and future therapies, Minerva medica, 107 (2016) 108-113.

[3] B. V. Zlokovic. Neurovascular pathways to neurodegeneration in Alzheimer's disease and other disorders, Nature reviews. Neuroscience, 12 (2011) 723-738.

[4] Z. Zhao, A. R. Nelson, C. Betsholtz, B. V. Zlokovic. Establishment and Dysfunction of the Blood-Brain Barrier, Cell, 163 (2015) 1064-1078.

[5] D. A. Nation, M. D. Sweeney, A. Montagne, A. P. Sagare, L. M. D’Orazio, M. Pachicano, et al. T. L. S. Benzinger, A.M. Fagan, J. M. Ringman, L. S. Schneider, J. C. Morris, H. C. Chui, M. Law, A. W. Toga, B. V. Zlokovic, Bloodbrain barrier breakdown is an early biomarker of human cognitive dysfunction, Nature medicine, 25 (2019) 270276.

[6] F. L. Cardoso, D. Brites, M. A. Brito. Looking at the blood-brain barrier: molecular anatomy and possible investigation approaches, Brain research reviews, 64 (2010) 328-363.

[7] M. D. Sweeney, A. P. Sagare, B. V. Zlokovic. Blood-brain barrier breakdown in Alzheimer disease and other neurodegenerative disorders, Nat Rev Neurol, 14 (2018) 133-150.

[8] B. Maziuk, H. I. Ballance, B. Wolozin. Dysregulation of RNA Binding Protein Aggregation in Neurodegenerative Disorders, Front Mol Neurosci, 10 (2017) 89.

[9] A. Pascale, S. Govoni. The complex world of post-transcriptional mechanisms: is their deregulation a common link for diseases? Focus on ELAV-like RNA-binding proteins, Cellular and molecular life sciences: CMLS, 69 (2012) 501-517.

[10] S. Taniuchi, M. Miyake, K. Tsugawa, M. Oyadomari, S. Oyadomari. Integrated stress response of vertebrates is regulated by four elF2a kinases, Scientific reports, 6 (2016) 32886.

[11] N. Fontrodona, F. Aubé, J. B. Claude, H. Polvèche, S. Lemaire, L. C. Tranchevent, et al. D. Auboeuf, Interplay between coding and exonic splicing regulatory sequences, Genome research, 29 (2019) 711-722. 
[12] F. Cid-Samper, M. Gelabert-Baldrich, B. Lang, N. Lorenzo-Gotor, R. D. Ponti, L. Severijnen, et al. An Integrative Study of Protein-RNA Condensates Identifies Scaffolding RNAs and Reveals Players in Fragile X-Associated Tremor/Ataxia Syndrome, Cell reports, 25 (2018) 3422-3434.e3427.

[13] T. Liu, H. Sun, D. Zhu, X. Dong, F. Liu, X. Liang, et al. TRA2A Promoted Paclitaxel Resistance and Tumor Progression in Triple-Negative Breast Cancers via Regulating Alternative Splicing, Molecular cancer therapeutics, 16 (2017) 1377-1388.

[14] Y. Tan, X. Hu, Y. Deng, P. Yuan, Y. Xie, J. Wang. TRA2A promotes proliferation, migration, invasion and epithelial mesenchymal transition of glioma cells, Brain research bulletin, 143 (2018) 138-144.

[15] X. Li, Y. Xue, X. Liu, J. Zheng, S. Shen, C. Yang, et al. ZRANB2/SNHG20/FOXK1 Axis regulates Vasculogenic mimicry formation in glioma, J Exp Clin Cancer Res, 38 (2019) 68.

[16] M. T. Melissari, P. Grote. Roles for long non-coding RNAs in physiology and disease, Pflugers Archiv: European journal of physiology, 468 (2016) 945-958.

[17] W. Gong, Y. Su, Y. Liu, P. Sun, X. Wang. Long non-coding RNA Linc00662 promotes cell invasion and contributes to cancer stem cell-like phenotypes in lung cancer cells, Journal of biochemistry, 164 (2018) 461469.

[18] Z. Liu, Y. Yao, S. Huang, L. Li, B. Jiang, H. Guo, et al. LINC00662 promotes gastric cancer cell growth by modulating the Hippo-YAP1 pathway, Biochemical and biophysical research communications, 505 (2018) 843849.

[19] L. Xie. MKL1/2 and ELK4 co-regulate distinct serum response factor (SRF) transcription programs in macrophages, BMC genomics, 15 (2014) 301.

[20] A. Fernández-Alvarez, M. Soledad Alvarez, C. Cucarella, M. Casado. Characterization of the human insulininduced gene 2 (INSIG2) promoter: the role of Ets-binding motifs, The Journal of biological chemistry, 285 (2010) 11765-11774.

[21] B. W. Day, B. W. Stringer, M. D. Spanevello, S. Charmsaz, P. R. Jamieson, K. S. Ensbey, et al. ELK4 neutralization sensitizes glioblastoma to apoptosis through downregulation of the anti-apoptotic protein $\mathrm{Mcl}-1$, Neuro-oncology, 13 (2011) 1202-1212.

[22] F. Tinarelli, C. Garcia-Garcia, F. Nicassio, V. Tucci. Parent-of-origin genetic background affects the transcriptional levels of circadian and neuronal plasticity genes following sleep loss, Philosophical transactions of the Royal Society of London. Series B, Biological sciences, 369 (2014) 20120471.

[23] E. Park, L. E. Maquat. Staufen-mediated mRNA decay, Wiley interdisciplinary reviews. RNA, 4 423-435.

[24] H. Cho, K. M. Kim, S. Han, J. Choe, S. G. Park, S. S. Choi, et al. Staufen1-mediated mRNA decay functions in adipogenesis, Molecular cell, 46 (2012) 495-506.

[25] S. Paul, W. Dansithong, K. P. Figueroa, D. R. Scoles, S. M. Pulst. Staufen1 links RNA stress granules and autophagy in a model of neurodegeneration, Nat Commun, 9 (2018) 3648.

Page $15 / 29$ 
[26] W. Liu, P. Wang, C. Shang, L. Chen, H. Cai, J. Ma, et al. Endophilin-1 regulates blood-brain barrier permeability by controlling ZO-1 and occludin expression via the EGFR-ERK1/2 pathway, Brain Res, 1573 (2014) 17-26.

[27] R. Su, S. Cao, J. Ma, Y. Liu, X. Liu, J. Zheng, et al. Knockdown of SOX20T inhibits the malignant biological behaviors of glioblastoma stem cells via up-regulating the expression of miR-194-5p and miR-122, Molecular cancer, 16 (2017) 171.

[28] A. R. Nelson, M. D. Sweeney, A. P. Sagare, B. V. Zlokovic. Neurovascular dysfunction and neurodegeneration in dementia and Alzheimer's disease, Biochim. Biophys. Acta, 1862 (2016) 887-900.

[29] A. Kurz, R. Perneczky. Amyloid clearance as a treatment target against Alzheimer's disease, Journal of Alzheimer's disease: JAD, 24 Suppl 2 (2011) 61-73.

[30] R. Ricciarelli, E. Fedele. The Amyloid Cascade Hypothesis in Alzheimer's Disease: It's Time to Change Our Mind, Current neuropharmacology, 15 (2017) 926-935.

[31] A. Carrano, J. J. Hoozemans, S. M. van der Vies, J. van Horssen, H. E. de Vries, A. J. Rozemuller. Neuroinflammation and blood-brain barrier changes in capillary amyloid angiopathy, Neuro-degenerative diseases, 10 (2012) 329-331.

[32] C. D. Pereira, F. Martins, J. Wiltfang, E. S. O. A. B. da Cruz, S. Rebelo. ABC Transporters Are Key Players in Alzheimer's Disease, Journal of Alzheimer's disease: JAD, 61 (2018) 463-485.

[33] S. Shen, H. Yu, X. Liu, Y. Liu, J. Zheng, P. Wang, et al. PIWIL1/piRNA-DQ593109 Regulates the Permeability of the Blood-Tumor Barrier via the MEG3/miR-330-5p/RUNX3 Axis, Molecular therapy. Nucleic acids, 10 (2018) 412-425.

[34] L. Zhu, M. Lin, J. Ma, W. Liu, L. Gao, S. Wei, et al. The role of LINC00094/miR-224-5p (miR-4975p)/Endophilin-1 axis in Memantine mediated protective effects on blood-brain barrier in AD microenvironment, J Cell Mol Med, 23 (2019) 3280-3292.

[35] A. Carrano, J. J. Hoozemans, S. M. van der Vies, A. J. Rozemuller, J. van Horssen, H. E. de Vries. Amyloid Beta induces oxidative stress-mediated blood-brain barrier changes in capillary amyloid angiopathy, Antioxidants \& redox signaling, 15 (2011) 1167-1178.

[36] S. Shang, Y. M. Yang, H. Zhang, L. Tian, J. S. Jiang, Y. B. Dong, et al. Intracerebral GM-CSF contributes to transendothelial monocyte migration in APP/PS1 Alzheimer's disease mice, Journal of cerebral blood flow and metabolism: official journal of the International Society of Cerebral Blood Flow and Metabolism, 36 (2016) 1978-1991.

[37] H. B. Dodiya, C. B. Forsyth, R. M. Voigt, P. A. Engen, J. Patel, M. Shaikh, et al. Chronic stress-induced gut dysfunction exacerbates Parkinson's disease phenotype and pathology in a rotenone-induced mouse model of Parkinson's disease, Neurobiology of disease, (2018). 
[38] E. F. Goodall, C. Wang, J. E. Simpson, D. J. Baker, D. R. Drew, P. R. Heath, et al. Age-associated changes in the blood-brain barrier: comparative studies in human and mouse, Neuropathology and applied neurobiology, 44 (2018) 328-340.

[39] C. Scheckel, E. Drapeau, M. A. Frias, C. Y. Park, J. Fak, I. Zucker-Scharff, et al. Regulatory consequences of neuronal ELAV-like protein binding to coding and non-coding RNAs in human brain, eLife, 5 (2016).

[40] M. Kamelgarn, J. Chen, L. Kuang, H. Jin, E. J. Kasarskis, H. Zhu. ALS mutations of FUS suppress protein translation and disrupt the regulation of nonsense-mediated decay, Proc Natl Acad Sci U S A, 115 (2018) E11904-E11913.

[41] R. G. de Bruin, E. P. van der Veer, J. Prins, D. H. Lee, M. J. Dane, H. Zhang, et al. The RNA-binding protein quaking maintains endothelial barrier function and affects VE-cadherin and $\beta$-catenin protein expression, Scientific reports, 6 (2016) 21643.

[42] Z. He, X. Ruan, X. Liu, J. Zheng, Y. Liu, L. Liu, et al. FUS/circ_002136/miR-138-5p/SOX13 feedback loop regulates angiogenesis in Glioma, Journal of experimental \& clinical cancer research: CR, 38 (2019) 65.

[43] Q. He, L. Zhao, X. Liu, J. Zheng, Y. Liu, L. Liu, et al. MOV10 binding circ-DICER1 regulates the angiogenesis of glioma via miR-103a-3p/miR-382-5p mediated ZIC4 expression change, Journal of experimental \& clinical cancer research: CR, 38 (2019) 9.

[44] T. Liu, H. Sun, D. Zhu, X. Dong, F. Liu, X. Liang, et al. TRA2A Promoted Paclitaxel Resistance and Tumor Progression in Triple-Negative Breast Cancers via Regulating Alternative Splicing, Mol Cancer Ther, 16 (2017) 1377-1388.

[45] P. Wu, X. Zuo, H. Deng, X. Liu, L. Liu, A. Ji. Roles of long noncoding RNAs in brain development, functional diversification and neurodegenerative diseases, Brain research bulletin, 97 (2013) 69-80.

[46] R. Johnson. Long non-coding RNAs in Huntington's disease neurodegeneration, Neurobiology of disease, 46 (2012) 245-254.

[47] S. Massone, I. Vassallo, G. Fiorino, M. Castelnuovo, F. Barbieri, R. Borghi, et al. A. Pagano, 17A, a novel noncoding RNA, regulates GABA B alternative splicing and signaling in response to inflammatory stimuli and in Alzheimer disease, Neurobiology of disease, 41 (2011) 308-317.

[48] S. Massone, E. Ciarlo, S. Vella, M. Nizzari, T. Florio, C. Russo, et al. NDM29, a RNA polymerase III-dependent non coding RNA, promotes amyloidogenic processing of APP and amyloid beta secretion, Biochimica et biophysica acta, 1823 (2012) 1170-1177.

[49] N. Khyzha, M. Khor, P. V. DiStefano, L. Wang, L. Matic, U. Hedin, et al. Regulation of expression in human vascular endothelial cells by a neighboring divergently transcribed long noncoding RNA, Proceedings of the National Academy of Sciences of the United States of America, (2019).

[50] Q. Lyu, S. Xu, Y. Lyu, M. Choi, C. K. Christie, O. J. Slivano, et al. stabilizes vascular endothelial cell adherens junctions through interaction with CKAP4, Proceedings of the National Academy of Sciences of the United 
States of America, 116 (2019) 546-555.

[51] P. Wu, Y. Gao, S. Shen, Y. Xue, X. Liu, X. Ruan, et al. KHDRBS3 regulates the permeability of blood-tumor barrier via cDENND4C/miR-577 axis, Cell death \& disease, 10 (2019) 536.

[52] M. J. Kang, K. Abdelmohsen, E. R. Hutchison, S. J. Mitchell, I. Grammatikakis, R. Guo, et al. HuD regulates coding and noncoding RNA to induce APP $\rightarrow$ A $\beta$ processing, Cell reports, 7 (2014) 1401-1409.

[53] J. E. Bestman, L. C. Huang, J. Lee-Osbourne, P. Cheung, H. T. Cline. An in vivo screen to identify candidate neurogenic genes in the developing Xenopus visual system, Developmental biology, 408 (2015) $269-291$.

[54] S. Kaikkonen, H. Makkonen, M. Rytinki, J. J. Palvimo. SUMOylation can regulate the activity of ETS-like transcription factor 4, Biochim. Biophys. Acta, 1799 (2010) 555-560.

[55] L. Jiang, J. Xiong, J. Zhan, F. Yuan, M. Tang, C. Zhang, et al. Ubiquitin-specific peptidase 7 (USP7)-mediated deubiquitination of the histone deacetylase SIRT7 regulates gluconeogenesis, The Journal of biological chemistry, 292 (2017) 13296-13311.

[56] A. V. Kossenkov, A. Vachani, C. Chang, C. Nichols, S. Billouin, W. Horng, et al. Resection of non-small cell lung cancers reverses tumor-induced gene expression changes in the peripheral immune system, Clinical cancer research: an official journal of the American Association for Cancer Research, 17 (2011) 5867-5877.

[57] B. S. Moon, J. Bai, M. Cai, C. Liu, J. Shi, W. Lu. Kruppel-like factor 4-dependent Staufen1-mediated mRNA decay regulates cortical neurogenesis, Nat Commun, 9 (2018) 401.

[58] C. Gong, L. E. Maquat. IncRNAs transactivate STAU1-mediated mRNA decay by duplexing with 3’ UTRs via Alu elements, Nature, 470 (2011) 284-288.

[59] T. P. Xu, X. X. Liu, R. Xia, L. Yin, R. Kong, W. M. Chen, et al. SP1-induced upregulation of the long noncoding RNA TINCR regulates cell proliferation and apoptosis by affecting KLF2 mRNA stability in gastric cancer, Oncogene, 34 (2015) 5648-5661.

\section{Tables}

Table A1. Primers used for RT-qPCR.

\begin{tabular}{lll}
\hline Primer or Probe & Gene & Sequence $\left(5^{\prime}->3^{\prime}\right)$ or Assay ID \\
\hline Primer & TRA2A & F: ATACTGGCAGCAGGGCAAAT \\
& & \\
& R: CGCCCAGTTCGCTGATCATA \\
& & F: GTCCTGCAGGCGTACAACTA \\
& ELK4 & R: CTTCTCGCTAGCAGGTGAGG \\
& F: GGGTTAGAATGGCACCCAC \\
& RAPDH & F: GCTGGACTTAGGGGAGCAAC \\
& & R: CTGGAAGATGGTGATTGGG \\
\end{tabular}


Table A2. shRNA target sequences

\begin{tabular}{|c|c|c|}
\hline Gene & & Sequence(5'->3') \\
\hline \multirow[t]{2}{*}{ TRA2A } & Sence & CACCGGCCTCAGTTTGTACACAACATTCAAGAGATGTTGTGTACAAACTGAGGCCTTTTTTG \\
\hline & Antisence & GATCCAAAAAAGGCCTCAGTTTGTACACAACATCTCTTGAATGTTGTGTACAAACTGAGGCC \\
\hline \multirow[t]{2}{*}{ LINC00662 } & Sence & TGCTAGCGAGAAGATAGCTTGGTTCAAGAGACCAAGCTATCTTCTCGCTAGCTTTTTTC \\
\hline & Antisence & TCGAGAAAAAAGCTAGCGAGAAGATAGCTTGGTCTCTTGAACCAAGCTATCTTCTCGCTAGCA \\
\hline \multirow[t]{2}{*}{ ELK4 } & Sence & CACCGGTGACTGTGAAAGTTTAAACTTCAAGAGAGTTTAAACTTTCACAGTCACCTTTTTTG \\
\hline & Antisence & GATCCAAAAAAGGTGACTGTGAAAGTTTAAACTCTCTTGAAGTTTAAACTTTCACAGTCACC \\
\hline \multirow[t]{2}{*}{ UPFI } & Sence & CACCGCGAGAAGGACTTCATCATCCTTCAAGAGACGATAACTCCTGAAATCCAGCTTTTTTG \\
\hline & Antisence & GATCCAAAAAAGCTGGATTTCAGGAGTTATCGTCTCTTGAAGGATGATGAAGTCCTTCTCGC \\
\hline \multirow[t]{2}{*}{ STAU1 } & Sence & CACCGCCGCAGGGAGTTTGTGATGCTTCAAGAGAGCATCACAAACTCCCTGCGGCTTTTTTG \\
\hline & Antisence & GATCCAAAAAAGCCGCAGGGAGTTTGTGATGCTCTCTTGAAGCATCACAAACTCCCTGCGGC \\
\hline
\end{tabular}

Table A3. Primers used for ChIP experiments

\begin{tabular}{|c|c|c|c|c|}
\hline Gene & $\begin{array}{l}\text { Binding site } \\
\text { or Control }\end{array}$ & Sequence $\left(5^{\prime}->3^{\prime}\right)$ & Product size (bp) & Annealing temperature $\left({ }^{\circ} \mathrm{C}\right)$ \\
\hline \multirow[t]{5}{*}{$\mathrm{ZO}-1$} & PCR1 & $\begin{array}{l}\text { F: CCAGTGTACTCCCTTTTATGGA } \\
\text { R: CCTGGAAGCTGAGGGACCTA }\end{array}$ & 110 & 51 \\
\hline & PCR2 & $\begin{array}{l}\text { F: GACTTGCTCTGTCACCCAGG } \\
\text { R: GAGTTCGAGACCAGCCCTG }\end{array}$ & 204 & 54 \\
\hline & PCR3 & F:TGCCCGGACTGCTTTGAAT & 257 & 54 \\
\hline & & R:AACAAAAACGAGACGCTGCC & & \\
\hline & PCR4 & F: GGCAGCGTCTCGTTTTTGTT & 111 & 52 \\
\hline \multirow[t]{3}{*}{ occludin } & PCR1 & $\begin{array}{l}\text { R: CAGGAGCTCCTTCTCAACCG } \\
\text { F: TCCCAGCTACTCAAGAGGCT } \\
\text { R: GCATGATCACTTGGGCTCCT }\end{array}$ & 222 & 53 \\
\hline & PCR2 & F: ACACAGTGTTAAGTGCAGACT & 207 & 54 \\
\hline & & R: TGGCCTGAGAAAAAGCACCT & & \\
\hline \multirow[t]{6}{*}{ Claudin-5 } & PCR1 & F: AGGATCCCTTGAGACCAGGA & 101 & 51 \\
\hline & & R: TATGCACCACCACCCACCTA & & \\
\hline & PCR2 & F: TACTAGAAAGGGGCTGGTGC & 205 & 57 \\
\hline & PCR3 & $\begin{array}{l}\text { R: ACTTGGAGTTTCCTGGCCAG } \\
\text { F: CACACTAGGTGAGCCAGACG }\end{array}$ & 229 & 56 \\
\hline & & R: TCAGTTCCATTGGACAGGGC & & \\
\hline & PCR4 & $\begin{array}{l}\text { F:CTGGCCAGGAAACTCCAAGT } \\
\text { R:GAGACAAAGGGACACGGAGG }\end{array}$ & 115 & 53 \\
\hline
\end{tabular}


Table A4. Antibodies for western blot

Primary antibodies:

TRA2A (1:500, Abcam, Cambridge, UK),

ELK4 (1:200, Santa Cruz Biotechnology, USA),

GAPDH (1:10000, Proteintech, USA),

ZO-1 (1:500, Life Technologies Corporation, MD, USA),

occludin1(1:600, Abcam, Cambridge, UK)

claudin-5 (1:300, Life Technologies Corporation, MD, USA).

Secondary antibodies:

HRP-linked anti-mouse IgG and HRP-linked anti-rabbit IgG antibodies

Figures

A

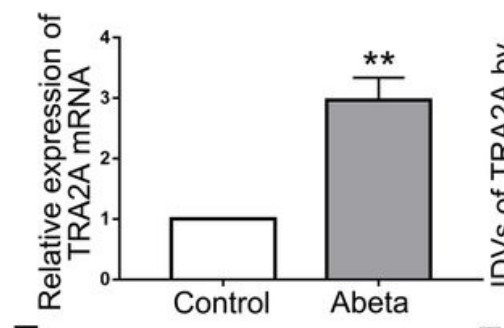

E

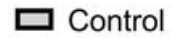

$\square$ shNC

$\square$ shTRA2A
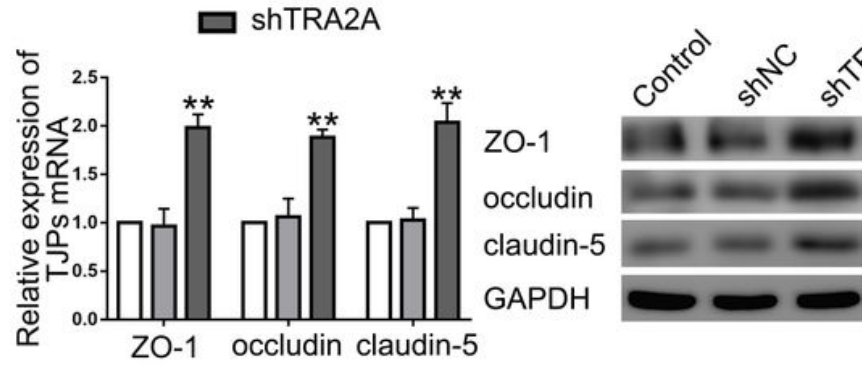

B

$\mathrm{F}$

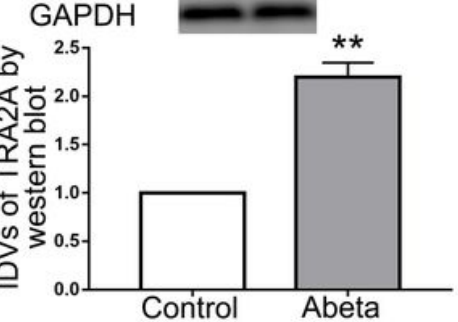

$\mathrm{F}$
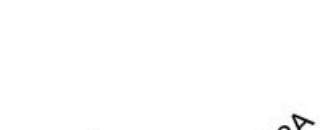

C

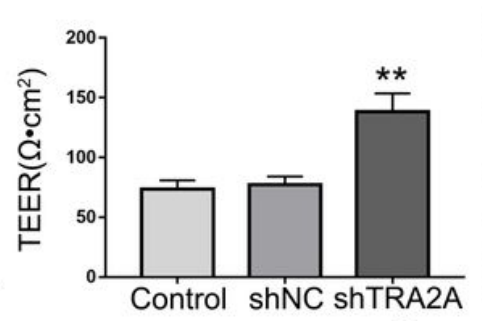

G

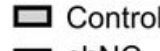
$\square$ shNC $\square$ shTRA2A

ZO-1

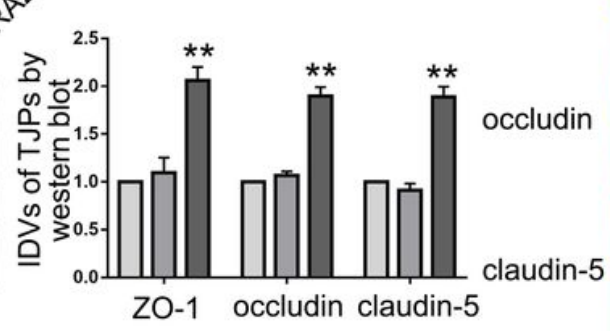

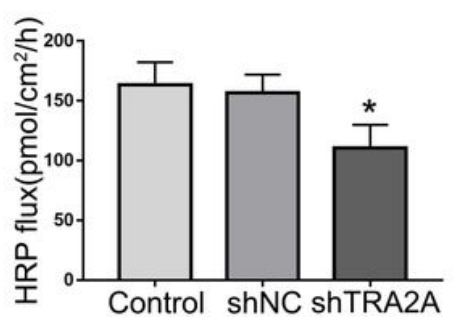

Control shNC shTRA2A

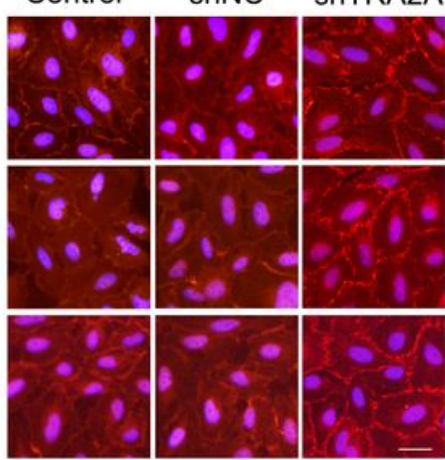

Figure 1 
TRA2A expression in AB1-42-incubated ECs and TRA2A regulated BBB permeability in AD microenvironment. (A) Relative mRNA expression of TRA2A in ECs pre-incubated with Abeta1-42 by qRT-PCR. Data are presented as mean $\pm S D$ ( $n=3$, each). ${ }^{*} P<0.01$ versus control group. (B) Relative TRA2A protein levels in ECs pre-incubated with Abeta1-42 by western blot. ${ }^{\star *} \mathrm{P}<0.01$ versus control group. ( $C$ and $\left.D\right)$ Effects of TRA2A on TEER values (C) and HRP flux (D). Data are presented as mean $\pm S D\left(n=3\right.$, each). ${ }^{*}<0.05$ versus shNC group. ${ }^{*} P<0.01$ versus shNC group. (E) Effects of TRA2A on ZO-1, occludin, and claudin-5 expression levels determined by qRTPCR. Data are presented as mean $\pm S D\left(n=3\right.$, each). ${ }^{*} P<0.01$ versus shNC group. (F) Effects of TRA2A on ZO1 , occludin, and claudin-5 expression levels determined by western blot. Data are presented as mean $\pm S D(n=3$, each). ${ }^{\star *} \mathrm{P}<0.01$ versus shNC group. (G) Effects of TRA2A on ZO-1, occludin, and claudin-5 expression levels and distribution determined by immunofluorescence staining ( $n=3$, each). ZO- 1 , occludin, and claudin- 5 (red) were labeled with secondary antibody against anti-ZO-1, anti-occludin, and anti-claudin-5 antibody, respectively, and nuclei (blue) were labeled with DAPI. Scale bar represents $30 \mu \mathrm{m}$. 

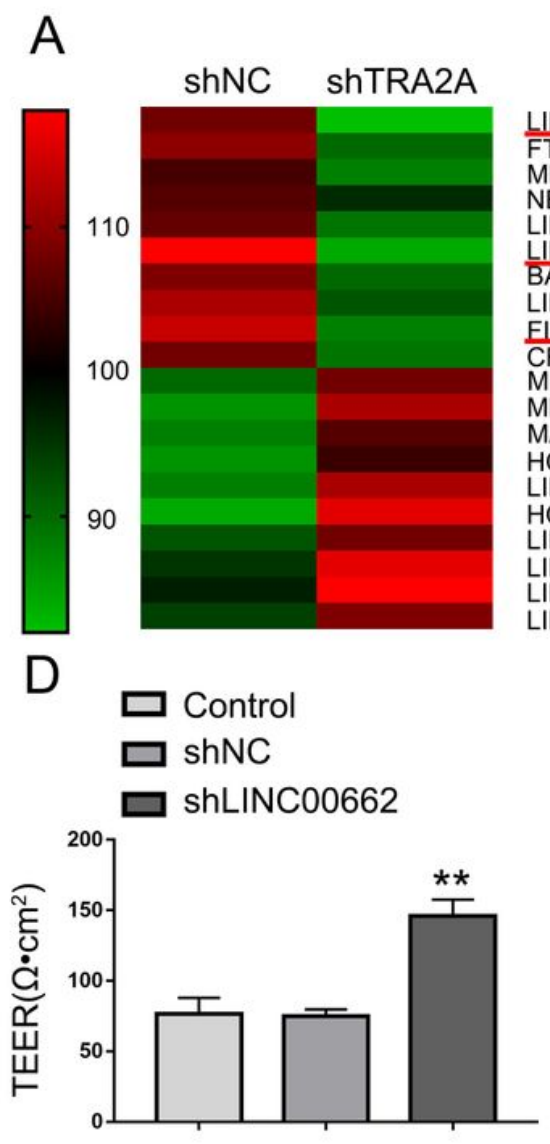

G

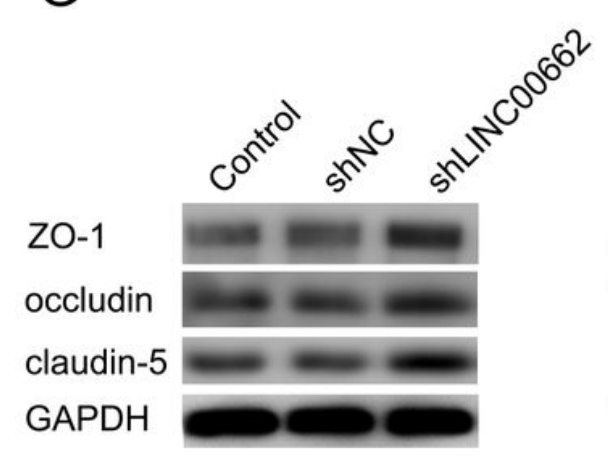

B
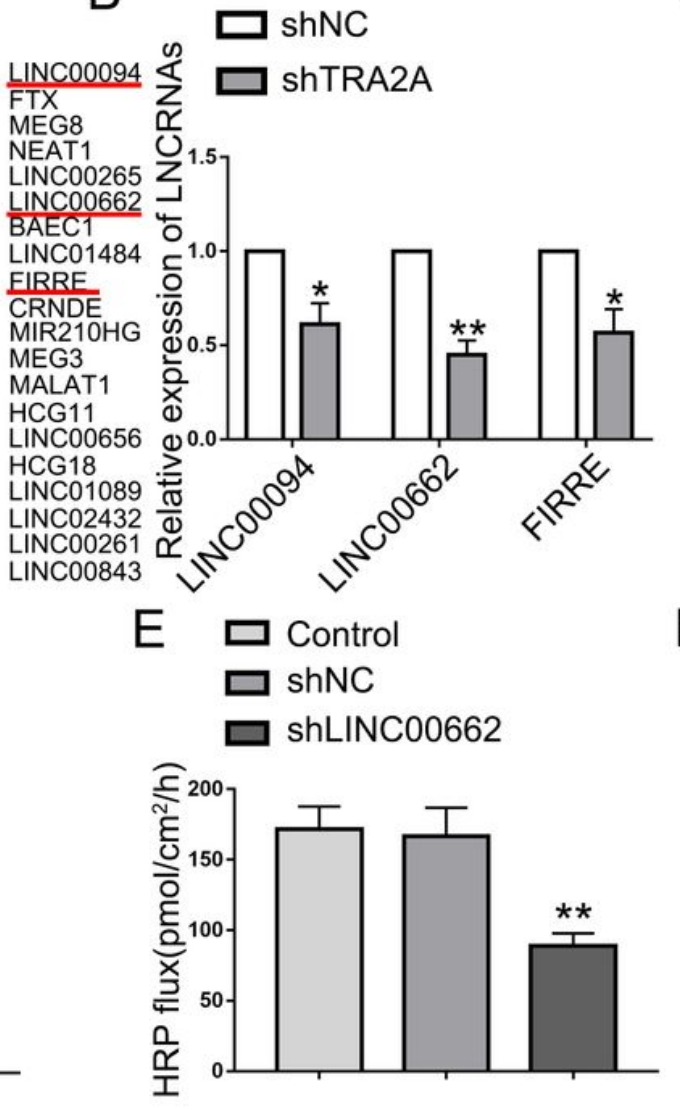

$\mathrm{F}$
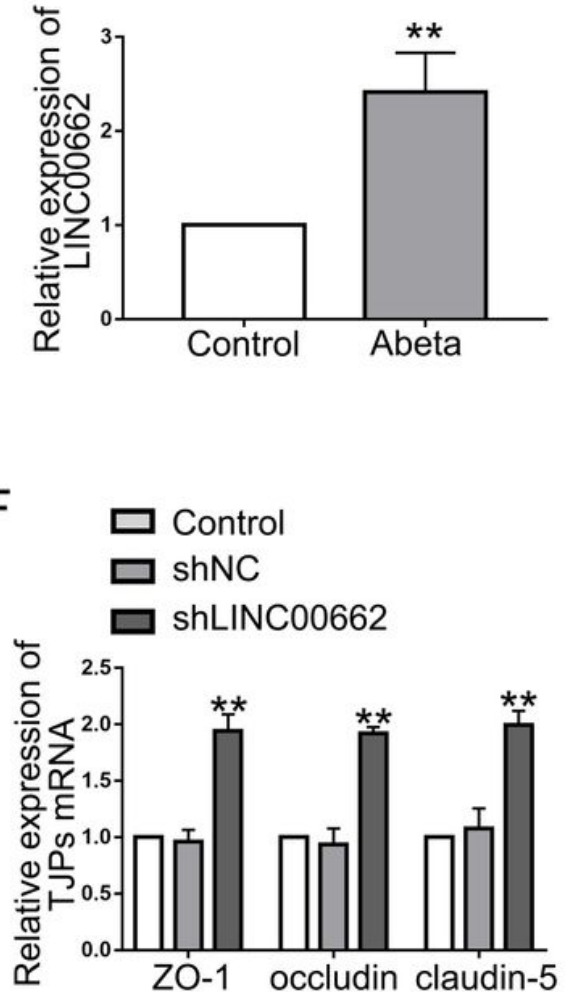

$\mathrm{H}$

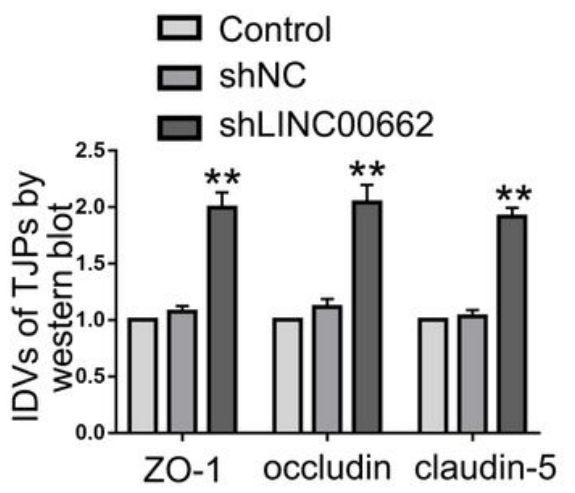

$\mathrm{ZO}-1$
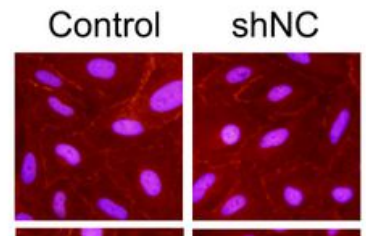

shLINC00662

occludin
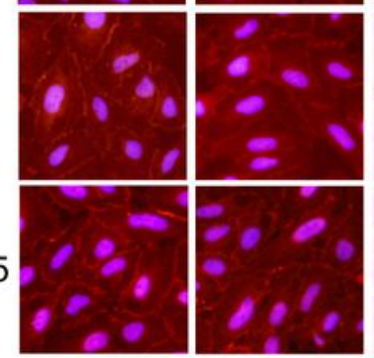

\section{Figure 2}

LINC00662 expression in AB1-42-incubated ECs and LINC00662 regulated BBB permeability in AD microenvironment. (A) LncRNA microarray analysis was performed in ECs treated with shTRA2A. Red indicates high relative expression and green indicates low relative expression. (B) Relative expression levels of LINC00094, LINC00662, and FIRRE determined by qRT-PCR. Data represent mean $\pm S D\left(n=3\right.$, each). ${ }^{*}<0.05$ versus shNC group. ${ }^{*} \mathrm{P}<0.01$ versus shNC group. (C) Relative expression of LINC00662 in ECs pre-incubated with $A \beta 1-42$ by qRT-PCR. Data are presented as mean $\pm S D\left(n=3\right.$, each). ${ }^{*} P<0.05$ versus control group. (D and E) Effects of LINC00662 on TEER values (D) and HRP flux (E). Data are presented as mean $\pm S D(n=3$, each). ${ }^{*} \mathrm{P}<0.01$ versus shNC group. (F) Effects of LINC00662 on ZO-1, occludin, and claudin- 5 expression levels determined by qRT-PCR. Data are presented as mean $\pm S D\left(n=3\right.$, each). ${ }^{*}<0.01$ versus shNC group. $(G)$ 
Effects of LINC00662 on ZO-1, occludin, and claudin-5 expression levels determined by western blot. Data are presented as mean $\pm S D\left(n=3\right.$, each). ${ }^{*} P<0.01$ versus shNC group. $(H)$ Effects of LINC00662 on ZO- 1 , occludin, and claudin- 5 expression levels and distribution determined by immunofluorescence staining ( $n=3$, each). ZO- 1 , occludin, and claudin-5 (red) were labeled with secondary antibody against anti-ZO-1, anti-occludin, and anticlaudin-5 antibody, respectively, and nuclei (blue) were labeled with DAPI. Scale bar represents $30 \mu \mathrm{m}$.

A
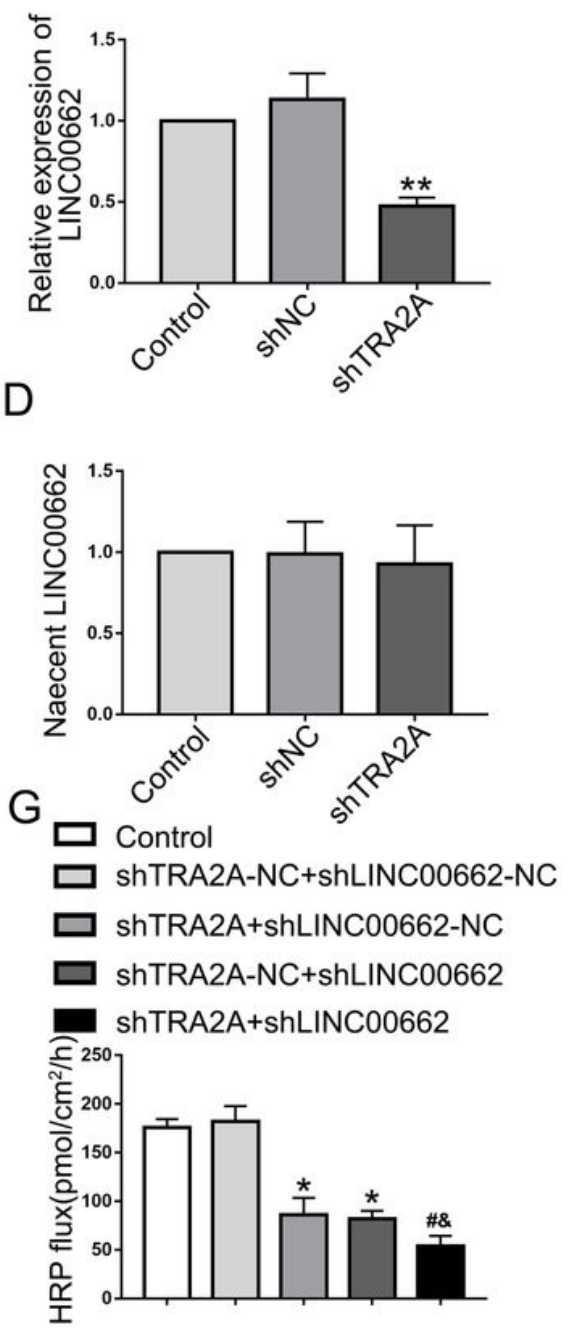

B

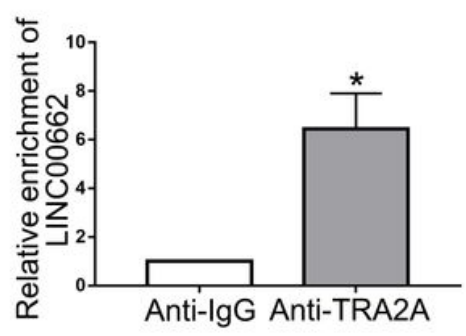

$\mathrm{E}$

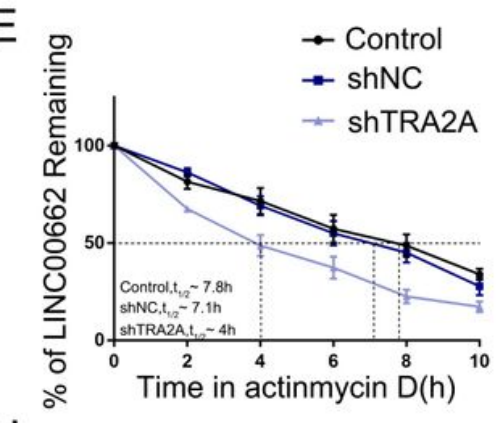

$\mathrm{H}$

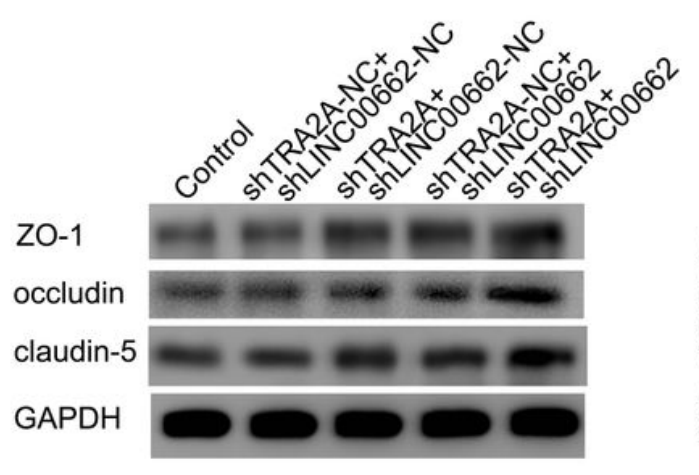

C

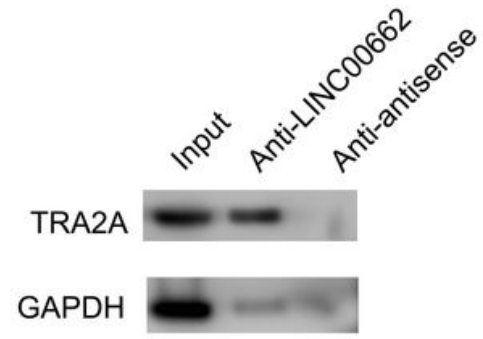

$\mathrm{F}$

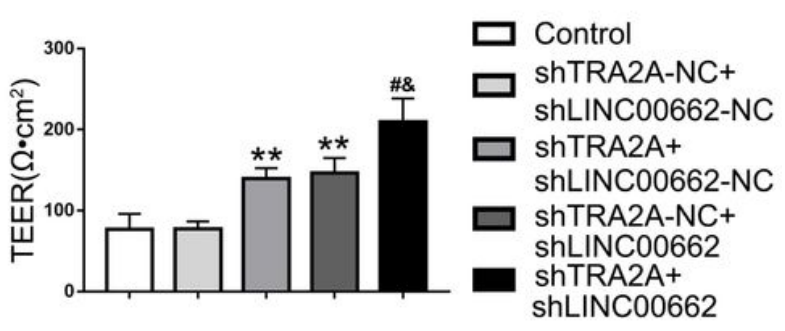

Figure 3

TRA2A konckdown decreased BBB permeability by stabilizing LINC00662. (A) Effects of TRA2A on LINC00662 expression determined by qRT-PCR. Data are presented as mean $\pm S D\left(n=4\right.$, each). ${ }^{\star} P<0.01$ versus shNC group. (B) RNA-IP confirmed the binding interaction between TRA2A and LINC00662. Relative enrichment was measured by qRT-PCR; Data are presented as mean $\pm S D\left(n=3\right.$, each). ${ }^{*} P<0.01$ versus Anti-IgG group. (C) RNA pull-down indicated that TRA2A bind to LINC00662 directly. (D) The graph shows nascent LINC00662 in AB1-42incubated ECs; data are presented as mean \pm SD ( $n=3$, each). (E) The graph shows LINC00662 levels at different times treated by ActD in the control group, shNC group, and shTRA2A group. ( $F$ and G) Effects of TRA2A and LINC00662 knockdown on TEER values (F) and HRP flux (G). Data are presented as mean $\pm S D(n=$ 3 , each). ${ }^{*} \mathrm{P}<0.01$ versus shTRA2A-NC + shLINC00662-NC group, $\# \mathrm{P}<0.05$ versus shTRA2A + shLINC00662NC, \&P $<0.05$ versus shTRA2A-NC + shLINC00662 group. (H) Effects of TRA2A and LINC00662 knockdown on 
ZO-1, occludin, and claudin-5 expression levels determined by western blot. Data are presented as mean \pm SD ( $\mathrm{n}$ $=3$, each). ${ }^{* * P}<0.01$ versus shTRA2A-NC + shLINC00662-NC group, \#\#P $<0.01$ versus shTRA2A + shLINC00662-NC, \&\&P $<0.01$ versus shTRA2A-NC + shLINC00662 group.
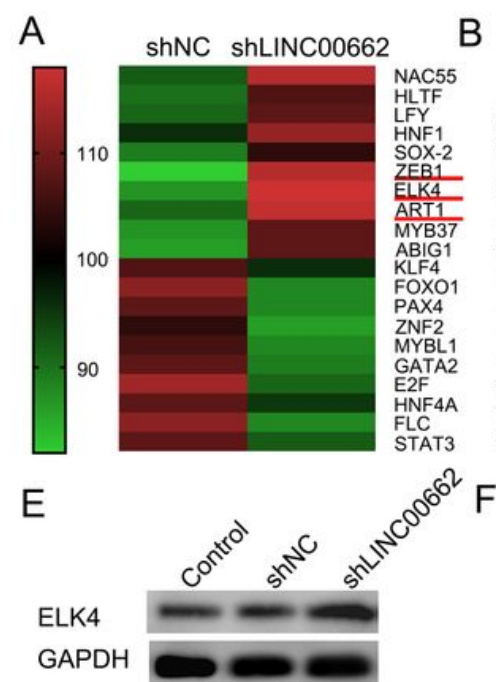

$\square$ Control

$\square$ shNC

口 shLINC00662

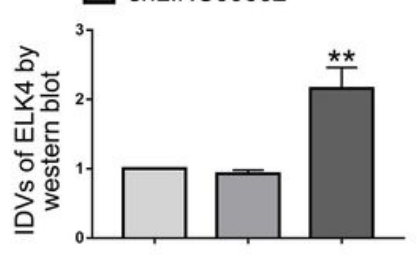

I
B
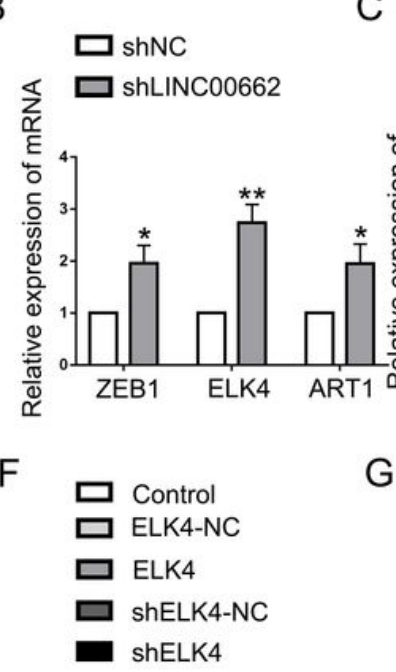

C

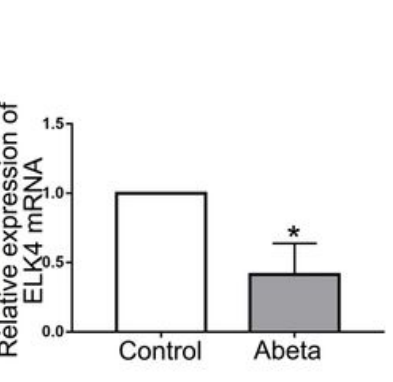

G

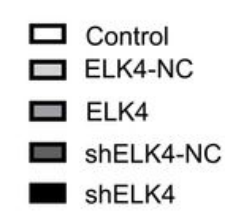

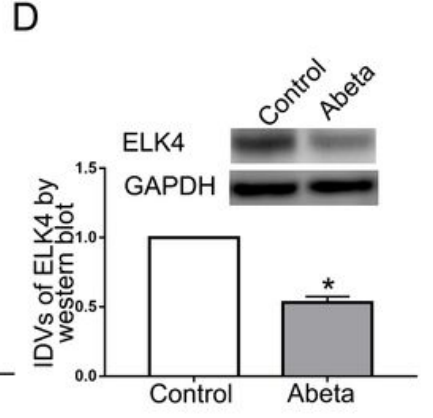

$\mathrm{H}$

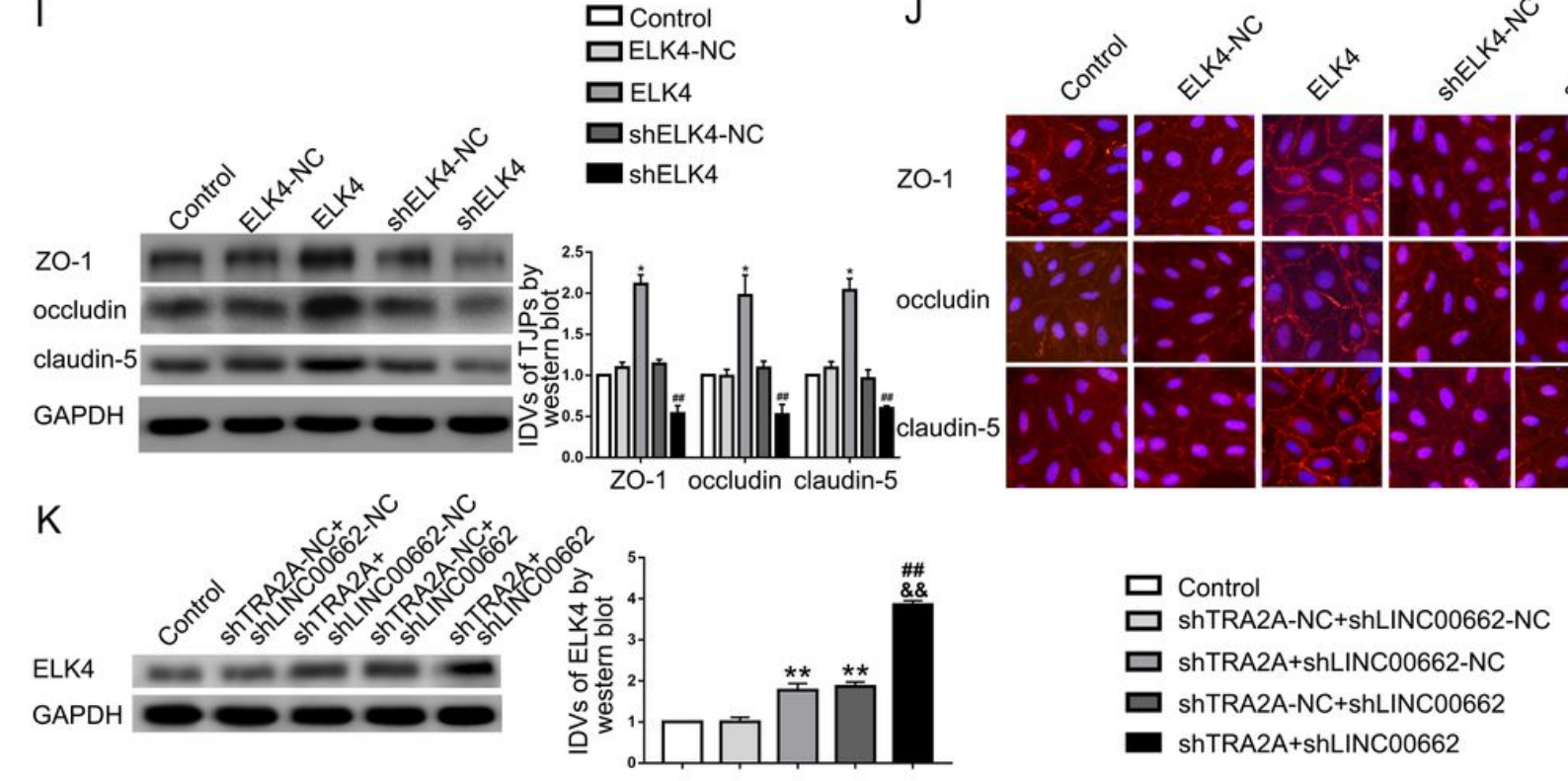

Figure 4

ELK4 expression in Aß1-42-incubated ECs and ELK4 regulated BBB permeability in AD microenvironment. (A) RNA microarray analysis was performed in ECs treated with shLINC00662. Red indicates high relative expression and green indicates low relative expression. (B) Relative expression levels of ZEB1, ELK4, and ART1 determined by qRT-PCR. Data represent mean $\pm S D\left(n=3\right.$, each). ${ }^{\star} P<0.01$ versus shNC group. (C) Effects of 
LINC00662 on ELK4 expression level in Aß1-42- incubated ECs by western blot. **P $<0.01$ versus control group. (D) Relative mRNA expression of ELK4 in ECs pre-incubated with AB1-42 by qRT-PCR. Data are presented as mean $\pm S D\left(n=4\right.$, each). ${ }^{*}<0.05$ versus control group. (E) Relative ELK4 protein levels in ECs pre-incubated with $A \beta 1-42$ by western blot. ${ }^{*}<0.05$ versus control group. ( $F$ and $G$ ) Effects of ELK4 on TEER values $(F)$ and HRP flux (G). Data are presented as mean $\pm S D\left(n=3\right.$, each). ${ }^{*} P<0.05$ versus ELK4-NC group. ${ }^{\star *} P<0.01$ versus ELK4-NC group. \#P $<0.05$ versus shELK4-NC group. \#\#P $<0.01$ versus shELK4-NC group. (H) Effects of ELK4 on ZO-1, occludin, and claudin-5 expression levels determined by qRT-PCR. Data are presented as mean $\pm S D(n=3$, each). *P $<0.05$ versus ELK4-NC group. \#\#P $<0.01$ versus shELK4-NC group. (I) Effects of ELK4 on ZO-1, occludin, and claudin- 5 expression levels determined by western blot. Data are presented as mean $\pm S D(n=3$, each). ${ }^{*} \mathrm{P}<0.05$ versus ELK4-NC group. \#\#P $<0.01$ versus shELK4-NC group. (J) Effects of ELK4 on ZO-1, occludin, and claudin-5 expression levels and distribution determined by immunofluorescence staining $(n=3$, each). ZO-1, occludin, and claudin-5 (red) were labeled with secondary antibody against anti-ZO-1, anti-occludin, and anti-claudin-5 antibody, respectively, and nuclei (blue) were labeled with DAPI. Scale bar represents $30 \mu \mathrm{m}$. (K) ELK4 expression regulated by TRA2A and LINC00662 knockdown; data are presented as mean \pm SD $(n=3$, each). ${ }^{\star *} \mathrm{P}<0.01$ versus shTRA2A-NC + shLINC00662-NC group, \#\#P $<0.01$ versus shTRA2A + shLINC00662-NC, $\& \& P<0.01$ versus shTRA2A-NC + shLINC00662 group. 
A

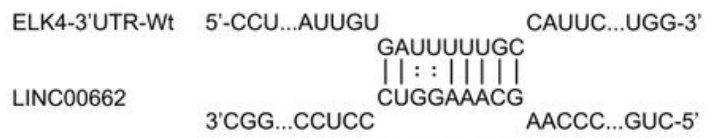

ELK4-3'UTR-MUt 5'-CCU...AUUGUAGUUGGGUAAACCC...GUC-5'

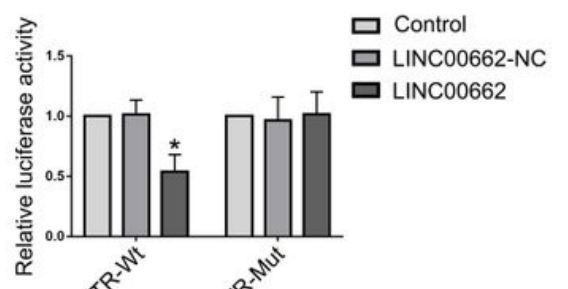

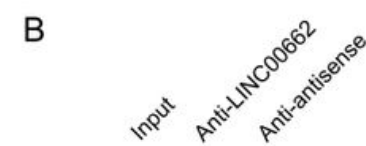

ELK4

GAPDH

E

$$
\text { ㅁ anti-lgG }
$$

$\square$ anti-STAU1
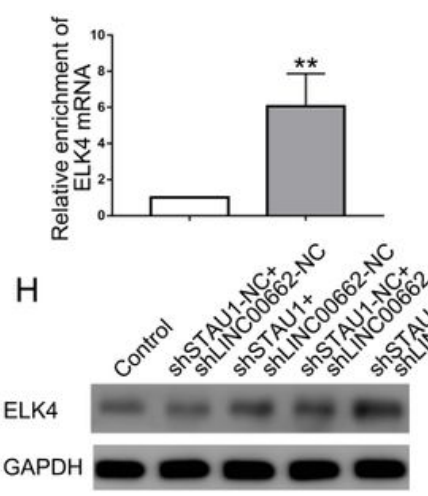

I

$\square$ Control

$\square$ shLINC00662-NC+ELK4-NC

$\square$ shLINC00662+ELK4

$\square$ shLINC00662-NC+shELK4-NC

- shLINC00662+shELK4

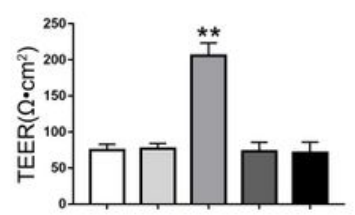

C

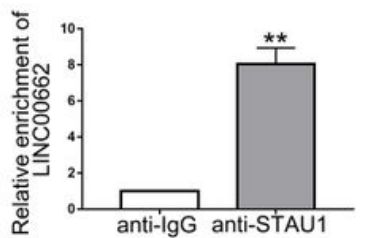

$\mathrm{F}$
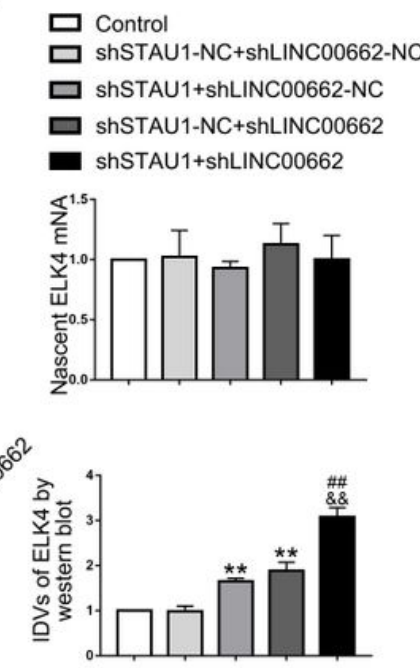

$\mathrm{J}$

$\square$ Control

$\square$ shLINC00662-NC+ELK4-NC

$\square$ shLINC00662+ELK4

$\square$ shLINC00662-NC+shELK4-NC

shLINC00662+shELK4

$\mathrm{K}$

ZO-1

occludin

claudin-5

GAPDH

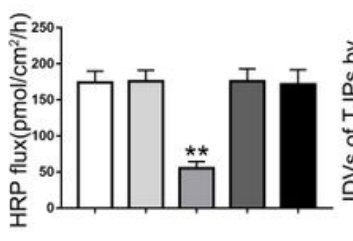

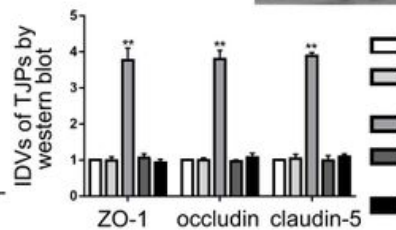

$\rightarrow$ Control

- shSTAU1-NC+shLINC00662-NC

- shSTAU1+shLINC00662-NC

* shSTAU1-NC+shLINC00662

$\rightarrow$ shSTAU1+shLINC00662

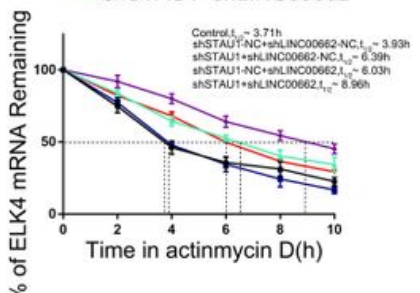

$\square$ Control

$\square$ shSTAU1-NC+shLINC00662-NC

$\square$ shSTAU1+shLINC00662-NC

$\square$ shSTAU1-NC+shLINC00662

shSTAU1+shLINC00662

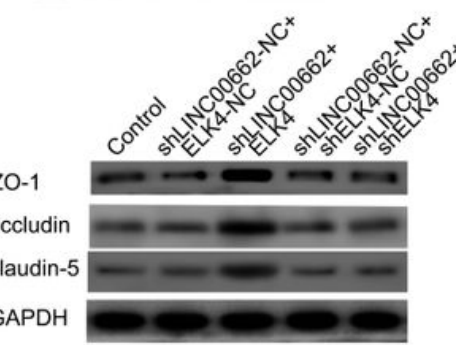

\section{Figure 5}

LINC00662 regulated BBB permeability by degrading ELK4 mRNA through SMD pathway. (A) The predicted LINC00662 binding site in ELK4 mRNA 3'UTR and results of dual-luciferase reporter assays. Data are presented as mean \pm SD ( $n=4$, each group). ${ }^{*} P<0.05$ versus ELK4-3'UTR-Wt + LINC00662-NC group. (B) RNA-pull down confirmed the binding interaction between LINC00662 and ELK4. (C) RNA-IP confirmed the binding interaction between LINC00662 and STAU1. Relative enrichment was measured by qRT-PCR; data are presented as mean \pm SD ( $n=3$, each). ${ }^{* * P}<0.01$ versus anti-IgG group. (D) RNA pull-down indicated that LINC00662 bind to STAU1 directly. (E) RNA-IP confirmed the binding interaction between ELK4 and STAU1. Relative enrichment was 
measured by qRT-PCR; data are presented as mean \pm SD $(n=3$, each). $* * P<0.01$ versus anti-lgG group. (F) The graph shows nascent ELK4 in Aß1-42-incubated ECs treated with shLINC00662 and shSTAU1; data are presented as mean \pm SD ( $n=3$, each). (G) Stability of ELK4 mRNA regulated by knockdown of LINC00662 and STAU1. (H) ELK4 expression regulated by STAU1 and LINC00662 knockdown; data are presented as mean \pm SD $\left(\mathrm{n}=3\right.$, each). ${ }^{\star *} \mathrm{P}<0.01$ versus shSTAU1-NC + shLINC00662-NC group, \#\#P $<0.01$ versus shSTAU1 + shLINC00662-NC, \&\&P < 0.01 versus shSTAU1-NC + shLINC00662 group. (I and J) TEER values (I) and HRP flux $(J)$ to evaluate the effects of LINC00662 and ELK4 on BBB integrity. Data are presented as mean \pm SD $(n=3$, each). ${ }^{* * P}<0.01$ versus shLINC00662-NC+ELK4-NC group. (K) Effects of LINC00662 and ELK4 on ZO-1, occludin, and claudin- 5 expression levels determined by western blot. Data are presented as mean \pm SD $(n=3$, each). ${ }^{* * P}<0.01$ versus shLINC00662-NC+ELK4-NC group.

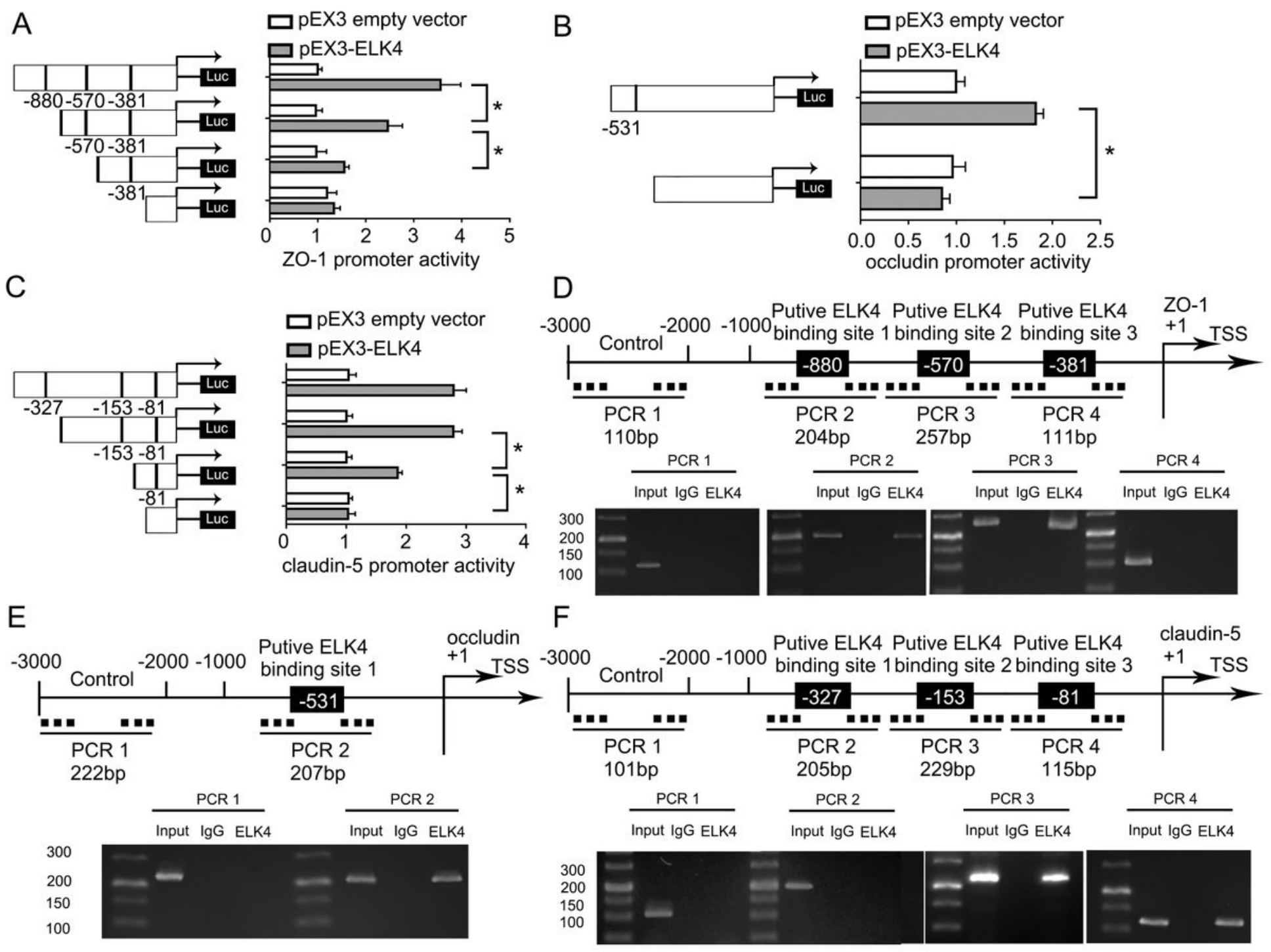

\section{Figure 6}

ELK4 increased the Promoter Activity of ZO-1, occludin, and claudin-5 (A-C) Schematic depiction of the different reporter plasmids and relative luciferase activity: ZO-1 (A), occludin (B), and claudin-5 (C) are shown. The Y-bar shows the deletion positions on the promoter fragments. The $\mathrm{X}$-bar shows the reporter vector activity after normalization with the cotransfected reference vector ( $\mathrm{pRL}-\mathrm{TK})$, and relative to the activity of the pEX3 empty vector, and the activity was set to 1 . Data represent mean $\pm S D\left(n=5\right.$, each). ${ }^{*} P<0.05$. (D-F) ELK4 bound to the 
promoters of ZO-1 (D), occludin (E), claudin-5 (F) in Aß1-42-incubated ECs. Transcription start site (TSS) was designated as +1 . Putative ELK4 binding sites are illustrated. Immunoprecipitated DNA was amplified by PCR. Normal rabbit IgG was used as a negative control.

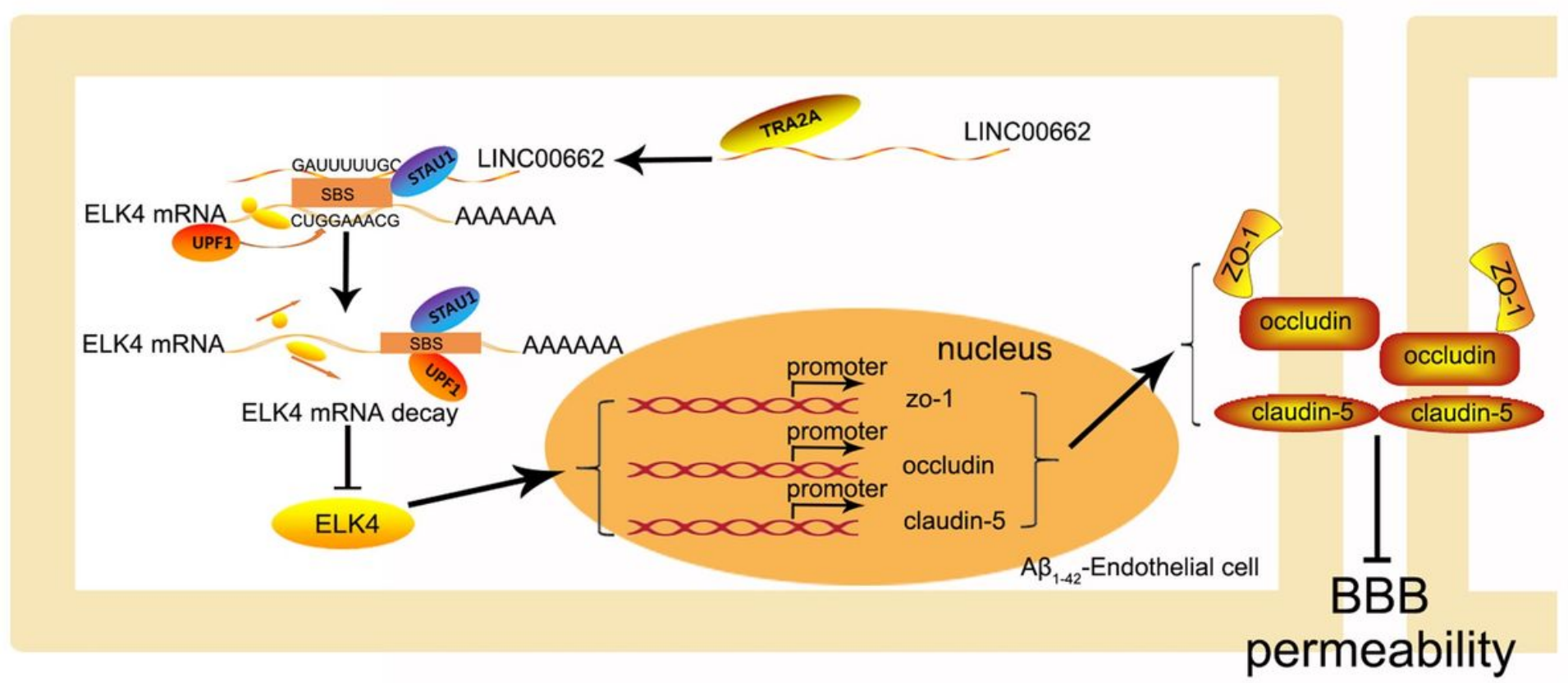

Figure 7

The schematic illusion of interactions between TRA2A, LINC00662 and ELK4 in Aß1-42-incubated ECs Supplementary Figure. (A) Relative expression of TRA2A evaluated using qRT-PCR in the TRA2A knockdown $A \beta 1-42$-incubated ECs; data are presented as mean $\pm S D(n=3)$. ${ }^{*} P<0.01$ versus sh-NC group. (B) Relative expression of TRA2A evaluated by western blot in the TRA2A knockdown AB1-42-incubated ECs; data are presented as mean $\pm S D(n=3)$. ${ }^{*} P<0.01$ versus sh-NC group. (C) Relative expression of LINC00662 evaluated using qRT-PCR in the LINC00662 knockdown Aß1-42-incubated ECs; data are presented as mean \pm SD $(n=3)$. $\star \star P<0.01$ versus sh-NC group. (D) The predicted combination of TRA2A and LINC00662 by starBase. (E and F) The Alu elements in LINC00662 and ELK4 3'UTR by RepeatMasker. (G) The predicted LINC00662 binding site in ELK4 mRNA 3'UTR by IntaRNA. (H) Relative expression of ELK4 evaluated using qRT-PCR in ELK4 knockdown and overexpression A 1-42-incubated ECs; data are presented as mean $\pm S D(n=3)$. **P $<0.01$ versus ELK4-NC group. \#\#P < 0.01 versus shELK4-NC. (I) Relative expression of ELK4 was evaluated by western blot in ELK4 knockdown and overexpression A 11-42-incubated ECs; data are presented as mean \pm SD $(n=3)$. ${ }^{*} P<0.01$ versus ELK4-NC group. \#\#P < 0.01 versus shELK4-NC. (J) Stability of ELK4 mRNA treatde with shUPF1; data are presented as mean \pm SD ( $n=3$, each group). (K) Effects of UPF1 on ELK4 expression; data are presented as mean $\pm S D\left(n=3\right.$, each group). ${ }^{\star} * P<0.01$ versus shNC group.

\section{Supplementary Files}

This is a list of supplementary files associated with this preprint. Click to download.

- FigureS.tif

- fulluneditedgelforFigure2.tif 
- fulluneditedgelforFigure4.tif

- WesternblotRawdata.xlsx

- fulluneditedgelforFigure3.tif

- fulluneditedgelforFigure5.tif

- Figure4J.tif

- Figure1G.tif

- Figure2H.tif

- fulluneditedgelforFigure1.tif 